\title{
Systematic Review \\ Active School Breaks and Students' Attention: A Systematic Review with Meta-Analysis
}

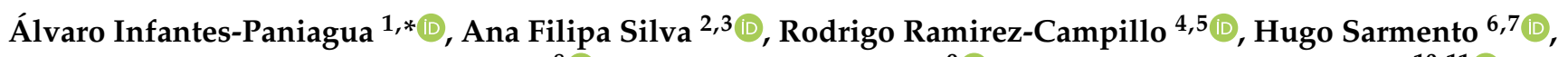 \\ Francisco Tomás González-Fernández ${ }^{8} \mathbb{(}$, Sixto González-Víllora ${ }^{9}\left(\mathbb{D}\right.$ and Filipe Manuel Clemente ${ }^{10,11}$
}

check for updates

Citation: Infantes-Paniagua, Á.; Silva, A.F.; Ramirez-Campillo, R.; Sarmento, H.; González-Fernández, F.T.; González-Víllora, S.; Clemente, F.M. Active School Breaks and Students' Attention: A Systematic Review with Meta-Analysis. Brain Sci. 2021, 11, 675. https://doi.org/ $10.3390 /$ brainsci11060675

Received: 19 April 2021

Accepted: 18 May 2021

Published: 21 May 2021

Publisher's Note: MDPI stays neutral with regard to jurisdictional claims in published maps and institutional affiliations.

Copyright: (C) 2021 by the authors Licensee MDPI, Basel, Switzerland. This article is an open access article distributed under the terms and conditions of the Creative Commons Attribution (CC BY) license (https:/ / creativecommons.org/licenses/by/ $4.0 /)$.
1 Department of Physical Education, Arts Education, and Music, Faculty of Education of Albacete, University of Castilla-La Mancha, 02071 Albacete, Spain

2 N2i, Polytechnic Institute of Maia, 4475-690 Maia, Portugal; anafilsilva@gmail.com

3 The Research Centre in Sports Sciences, Health Sciences and Human Development (CIDESD), 5001-801 Vila Real, Portugal

4 Department of Physical Activity Sciences, Universidad de Los Lagos, Santiago 8320000, Chile; r.ramirez@ulagos.cl

5 Centro de Investigación en Fisiología del Ejercicio, Facultad de Ciencias, Universidad Mayor, Santiago 7500000, Chile

6 University of Coimbra, 3004-531 Coimbra, Portugal; hg.sarmento@gmail.com

7 Research Unit for Sport and Physical Activity, Faculty of Sport Sciences and Physical Education, 3004-531 Coimbra, Portugal

8 Centro de Estudios Superiores Alberta Giménez, Department of Physical Activity and Sport Sciences, Pontifical University of Comillas, 07013 Palma, Spain; francis.gonzalez.fernandez@gmail.com

9 Department of Physical Education, Arts Education, and Music, Faculty of Education of Cuenca, University of Castilla-La Mancha, 17071 Cuenca, Spain; sixto.gonzalez@uclm.es

10 Escola Superior Desporto e Lazer, Instituto Politécnico de Viana do Castelo, Rua Escola Industrial e Comercial de Nun'Álvares, 4900-347 Viana do Castelo, Portugal; Filipe.clemente5@gmail.com

11 Instituto de Telecomunicações, Delegação da Covilhã, 1049-001 Lisboa, Portugal

* Correspondence: Alvaro.Infantes@uclm.es; Tel.: +34-967-599-200 (ext. 2564)

Abstract: School physical activity breaks are currently being proposed as a way to improve students' learning. However, there is no clear evidence of the effects of active school breaks on academicrelated cognitive outcomes. The present systematic review with meta-analysis scrutinized and synthesized the literature related to the effects of active breaks on students' attention. On January 12th, 2021, PubMed, PsycINFO, Scopus, SPORTDiscus, and Web of Science were searched for published interventions with counterbalanced cross-over or parallel-groups designs with a control group, including school-based active breaks, objective attentional outcomes, and healthy students of any age. Studies' results were qualitatively synthesized, and meta-analyses were performed if at least three study groups provided pre-post data for the same measure. Results showed some positive acute and chronic effects of active breaks on attentional outcomes (i.e., accuracy, concentration, inhibition, and sustained attention), especially on selective attention. However, most of the results were not significant. The small number of included studies and their heterogeneous design are the primary limitations of the present study. Although the results do not clearly point out the positive effects of active breaks, they do not compromise students' attention. The key roles of intensity and the leader of the active break are discussed. INPLASY registration number: 202110054.

Keywords: physical activity; exercise; attention; attentional bias; arousal; randomized controlled trials; non-randomized controlled trials; cross-over studies; systematic review; meta-analysis

\section{Introduction}

Active breaks (ABs) are currently gaining attention within the educational context [1] ABs consist of short periods (usually between five and $15 \mathrm{~min}$ ) of classroom-based physical activity (PA) [2], which are integrated into the routine of the class [1,3]. These can be implemented by the teacher [2] during or between academic instructions [4]. Compared to 
other kinds of school-based PA interventions, ABs show some advantages. For example, (i) they do not require special spaces or equipment, (ii) teachers can choose when to utilize ABs according to their lessons' necessities [2], and (iii) they are not too time-consuming for practical use [5].

Some authors consider ABs to be an effective approach to promote PA with the final aim of improving students' health since school time represents an ideal setting for such purposes [6]. There is evidence confirming that school-based PA interventions increase students' PA levels [7,8]. In fact, the scientific literature suggests that the brain learns better when active methodologies (active role of students) are implemented instead of passive methodologies or traditional lessons [9]. This is of special interest nowadays because most young students do not meet the PA guidelines recommended by the World Health Organization [10]. Additionally, there is extensive research showing that PA interventions can improve students' cognitive, metacognitive, and academic outcomes, such as working memory, attention, processing speed, and academic performance [11,12]. Both the acute and chronic effects of school-based PA interventions on cognitive and academic performance have been extensively reviewed $[1,3,12,13]$. Although their positive effects are not completely clear for all those variables (e.g., attention, processing speed, or academic performance), it seems that increasing the amount of school time spent on PA does not compromise students' cognitive or academic performance. Therefore, school-based PA interventions are promising practices when appropriately implemented [14].

Among the cognitive outcomes addressed, attention is of great relevance for students since it plays a key role in learning [15] and academic achievement [16]. Conceptualizing attention is not easy due to the myriad of concepts that it involves. Therefore, in the present study, following Janssen et al. (2014) [17], we did not focus on a single measure of attention but instead considered attentional outcomes objectively measured within AB research.

Some authors perceive attention as a process of exerting mental effort on specific stimuli [18], while for others, it is like a "gate" that manages the input of information into conscious awareness [6]. Notwithstanding, most researchers agree with the multicomponent nature of attention [19]. This is reflected in the numerous different tasks used to measure attention in previous research [17], such as concentration tasks, time-on-task behaviors, or even electroencephalography.

It has been hypothesized that the effects of PA on attention, both from acute and chronic points of view, have a physiological basis (e.g., cardiovascular hypothesis, intensity of PA, or increases in cerebral blood flow and the number of neurotransmitters) [20]. There is sufficient evidence to suggest that ABs improve cognition, especially attention [20-22]. Furthermore, previous evidence has shown that ABs can improve students' attention $[7,8,23]$. However, results are still heterogeneous [2] and require further confirmation [3].

The inconsistent results presented in the literature could perhaps be explained by differences in the factors considered from a study design, how attention is measured, or the inclusion of samples representing different age groups [6] or cardiovascular fitness levels [6], just as happens in research on overall cognitive performance [2,12]. Moreover, in one study [24], it was suggested that different results on cognitive effects arise from differences in $\mathrm{AB}$ characteristics (e.g., cognitive engagement or complexity, intensity, duration).

Regarding the acute effects of $\mathrm{AB}$ duration, a recent meta-regression analysis suggested that shorter PA bouts may be more effective than longer ones for improving attention [12]. However, other research has indicated that longer bouts (i.e., >20 min) showed greater effects [20]. Since other studies did not find differences regarding the duration of PA bout [25], this topic requires further research to investigate the optimal duration of ABs.

Researchers have already highlighted the importance of investigating the duration of the cognitive benefits that remain after a PA bout, which is difficult to establish since post-test timings vary widely across studies, and most studies do not correctly report this information [1].

Finally, the person responsible for delivering the $\mathrm{AB}$ might also influence the characteristics of PA, especially regarding the intensity and student engagement [26,27]. In 
their review, Daly-Smith et al. (2019) [1] reported that the highest proportion of time spent on moderate-vigorous physical activity (MVPA) in active lessons was associated with the researcher-led intervention. Similarly, Watson et al. (2017) [3] pointed out that programs presented a higher fidelity to the required intensity when research staff was responsible for the intervention. These studies highlight the importance of the intervention deliverers' qualifications, which has not yet been clarified in the AB literature.

For more than a decade, students' attention deficits have been a significant concern of teachers [28]. ABs seem to be a promising way to enhance students' attentional levels in the class. However, several questions remain to be answered. To the best of our knowledge, there is only one previous meta-analysis that examined this issue [2], and it focused only on overall cognitive- or academic-related outcomes in 6- to 9-year-old students.

Therefore, the aim of this systematic review with meta-analysis was to scrutinize and synthesize the literature related to the effects of $\mathrm{ABs}$ (compared to control conditions) on the attention of students (of any age). We also addressed some possible moderators that previous research pointed out as relevant to the effects of $A B s$ on cognition.

\section{Materials and Methods}

This systematic review (with meta-analysis) followed established international guidelines $[29,30]$. The protocol was published in INPLASY (International Platform of Registered Systematic Review and Meta-analysis Protocols) with the identification number of 202,110,054 and DOI 10.37766/inplasy2021.1.0054.

\subsection{Eligibility Criteria}

According to the Participants, Intervention, Comparators, Outcomes, and Study design (P.I.C.O.S.) approach, the inclusion and exclusion criteria for this systematic review and meta-analysis can be found in Table 1 .

Table 1. Inclusion and exclusion criteria following the P.I.C.O.S. approach.

\begin{tabular}{|c|c|c|}
\hline PICOS & Inclusion Criteria & Exclusion Criteria \\
\hline Population & $\begin{array}{l}\text { Healthy students of any age and of any sex from } \\
\text { elementary to college educational levels. }\end{array}$ & $\begin{array}{c}\text { Populations other than students (e.g., workers, } \\
\text { athletes). Students with a diagnosed } \\
\text { mental disease. }\end{array}$ \\
\hline Intervention & $\begin{array}{l}\text { ABs consisting of short bouts of exercise in class } \\
\text { during or between academic lessons (e.g., } \\
\text { structured exercises, free exercise). }\end{array}$ & $\begin{array}{l}\text { No ABs (e.g., physical education classes; playing } \\
\text { with instruments without allowing PA). }\end{array}$ \\
\hline Comparator & $\begin{array}{c}\text { Control conditions (passive or non-active breaks } \\
\text { with limited PA). }\end{array}$ & $\begin{array}{l}\text { Other forms of physical activity interventions } \\
\text { (e.g., physical education lessons). }\end{array}$ \\
\hline Outcome & $\begin{array}{c}\text { Attentional outcomes (e.g., focused or selective } \\
\text { attention, vigilance, inhibitory control) measured } \\
\text { before (pre-) and after (post-) ABs or a chronic } \\
\text { intervention of ABs. }\end{array}$ & $\begin{array}{l}\text { Outcomes other than attention. No pre-post } \\
\text { comparison. Inaccessible pre- or } \\
\text { post-intervention data. }\end{array}$ \\
\hline Study design & $\begin{array}{c}\text { Counterbalanced cross-over design and } \\
\text { parallel-groups design. }\end{array}$ & $\begin{array}{l}\text { Study designs that do not allow within-subjects } \\
\text { comparisons for both control and } A B \text { conditions. } \\
\text { Non-original articles (e.g., reviews, letters to }\end{array}$ \\
\hline Additional criteria & Original and full-text studies written in English. & $\begin{array}{l}\text { editors, trial registrations, proposals for } \\
\text { protocols, editorials, book chapters, } \\
\text { conference abstracts). }\end{array}$ \\
\hline
\end{tabular}

\subsection{Information Sources}

Five electronic databases (PubMed, PsycINFO, Scopus, SPORTDiscus, and Web of Science) were searched for relevant publications prior to 12 January 2021. Keywords and synonyms were entered in various combinations: ("activ* break" OR "physical break" OR "physical activity break" OR "exercise break" OR "brain break" OR "brain hacking" OR "movement learning" OR "active learning") AND (student* OR class* OR school*) AND attent*. Additionally, the reference lists of included studies were manually searched 
to identify potentially eligible studies not captured by the electronic searches. All records were screened by two researchers (AIP and FTGF).

\subsection{Data Extraction}

A data extraction was prepared in Microsoft Excel sheet (Microsoft Corporation, Readmon, WA, USA), similar to the Cochrane Consumers and Communication Review Group's data extraction template (Group, 2016). The Excel sheet was used to assess inclusion and exclusion requirements and subsequently tested for all selected studies. The process was independently conducted by two authors (AIP and HS). Any disagreement regarding study eligibility was resolved in a discussion with a third author (FTGF) when necessary. Full text articles excluded and the reasons for doing so were recorded (see Table A1 in Appendix A). All the records were stored in the sheet.

\subsection{Data Items}

The following categories of information were extracted from included articles: (i) randomization unit, design, number of participants (n), age group (schoolchildren, young adults or both), sex (men, women or both); (ii) fitness of participants; (iii) identification of ABs (time, duration, weekly and/or daily frequency, intensity and type of PA, academic content, the person who is responsible for the $A B$, and protocol), (iv) treatment fidelity, (v) measurement of attention (i.e., task), (vi) time of measurements (pre and post) and (vii) effect measured (i.e., acute effects vs. chronic effects).

\subsection{Assessment of Methodological Quality}

The methodological quality of studies was assessed using the Revised Cochrane risk-ofbias tool for randomized trials (RoB 2) for randomized controlled trials (RCTs) [31], as well as the supplements for cluster randomized trials (CRTs) [32] and for cross-over trials [33]. For non-RCT, the Cochrane risk of bias tool for non-randomized studies of interventions (ROBINS-I) scale was used [34]. These tools include a minimum of 21 items that enable the assessment of the risk of bias (i.e., "low risk", "some concerns", or "high risk") of several dimensions that vary according to the study design (namely, bias arising from the randomization process, bias due to deviations from intended interventions, bias due to missing outcome data, bias in measurement of the outcome, and bias in selection of the reported result). An 'intention-to-treat' effect approach was followed for all the assessments, which implies that the interest focused on the effect of assignment to the interventions. This approach was followed because there was a wide variety of study designs and protocols could not be reviewed in most of the cases. Altogether an overall level of risk of bias per study was computed. Risk of bias assessments were based on the published articles, which were accompanied with the trial protocols in two studies [6,35]. Two of the authors (AIP and HS) independently screened and assessed the included articles. Discrepancies were solved by consensus between the two authors without the need for assistance from a third author.

\subsection{Statistical Analyses}

Meta-analyses were performed if at least three study groups provided pre-post ABrelated data for the same measure. Using a random-effects model, the means and standard deviations (SD) for dependent variables were used to calculate effect sizes (ES; Hedges' g) for each outcome in $\mathrm{AB}$ treatments and control conditions. When means and SDs were not available, they were obtained from $95 \%$ confidence intervals (CIs) or standard error of mean (SEM), using Cochrane recommended formulas. Data were standardized using postintervention SD values. The ES values are presented with 95\% confidence intervals (CI). Calculated ES were interpreted using the following scale: $<0.2$, trivial; $0.2-0.6$, small; $>0.6-$ 1.2 , moderate; $>1.2-2.0$, large; $>2.0-4.0$, very large; $>4.0$, extremely large [36]. Heterogeneity was assessed using the $I^{2}$ statistic, with values of $<25 \%, 25-75 \%$, and $>75 \%$ considered to represent low, moderate, and high levels of heterogeneity, respectively [37]. The risk 
of bias was explored using the extended Egger's test [38]. To adjust for publication bias, a sensitivity analysis was conducted using the trim and fill method [39], with L0 as the default estimator for the number of missing studies [40]. All analyses were carried out using the Comprehensive Meta-Analysis software (version 2; Biostat, Englewood, NJ, USA). Statistical significance was set at $p \leq 0.05$.

\section{Results}

\subsection{Study Identification and Selection}

The database search retrieved 1809 titles, which were exported to reference manager software (EndNoteTM X9, Clarivate Analytics, Philadelphia, PA, USA). Duplicates (520 references) were subsequently removed either automatically or manually. The remaining 1289 articles were screened for their relevance based on titles and abstracts, resulting in the removal of a further 1244 studies. Following the screening procedure, 45 articles were selected for in-depth reading and analysis. After reading full texts, a further 36 studies were excluded due to not meeting the eligibility criteria (Table A1). Finally, nine studies were selected for the further analysis together with another seven studies that were identified from other sources, reaching a total of 16 included studies (Figure 1), involving 3383 participants between 6 and 13 years old. Due to the limited number of studies included into the review for each attentional outcome (e.g., global attention, selective attention, inhibition, etc.), results from participants of all ages included were grouped together despite this age range involves different stages of development.

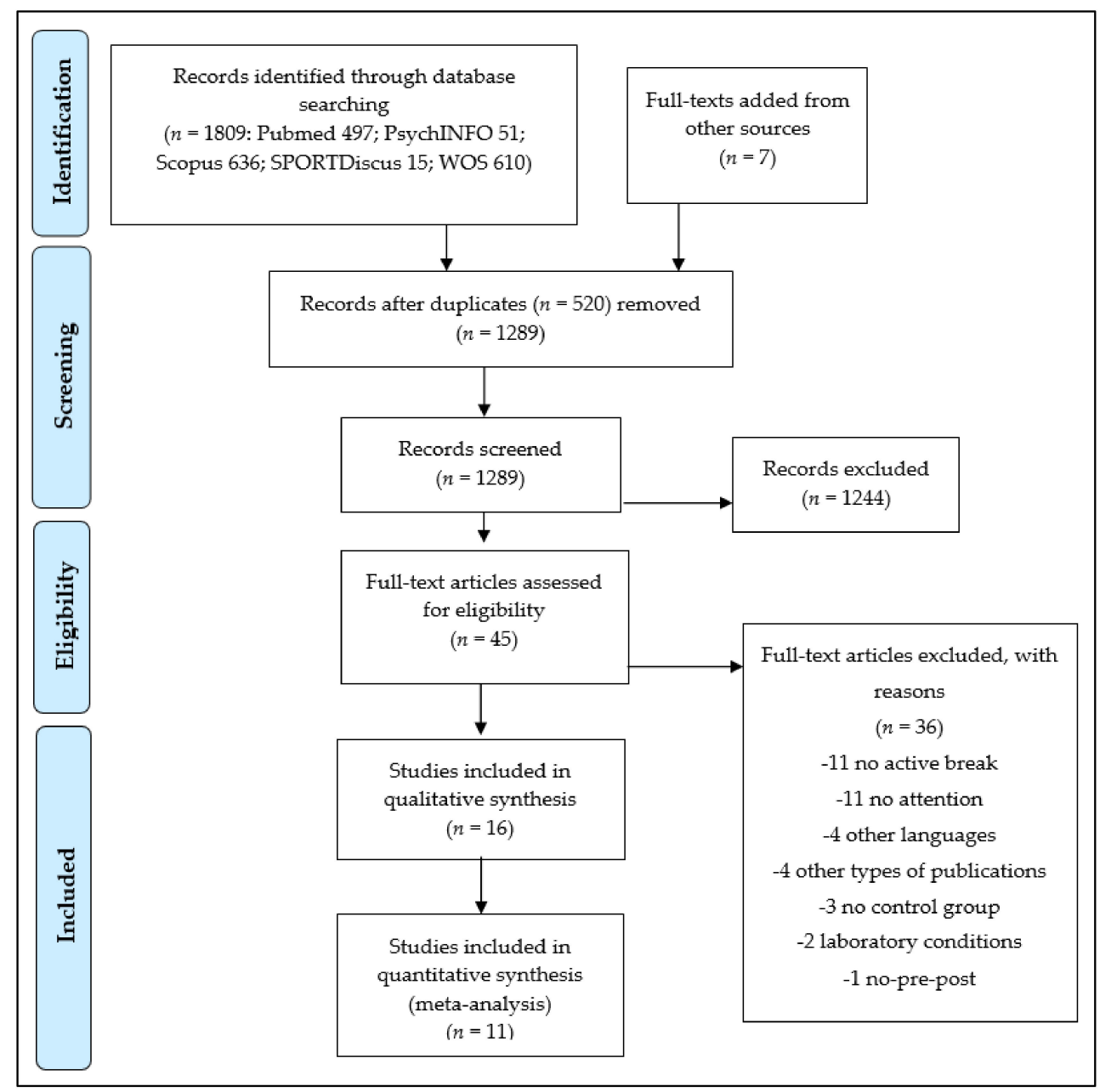

Figure 1. Preferred Reporting Items for Systematic Reviews and Meta-Analyses (PRISMA) flow diagram highlighting the selection process for the studies included in the systematic review. 


\subsection{Study Characteristics}

Eleven studies followed a parallel-groups design with seven RCTs [19,35,41-45], three CRTs [24,46,47], and one non-randomized [48] (Table 2). Additionally, the remaining five studies followed a cross-over design (three single-group design studies $[6,23,49]$ and two CRTs [50,51]) (Table 3).

The protocols' characteristics for all studies can be found in Table 4. Most of the studies addressed the acute effects of ABs [6,19,23,35,41-45,49-51], while only four addressed chronic effects $[24,46-48]$. Considering both types of effects, $A B$ duration varied from 4 [23] to $\approx 25 \mathrm{~min}$ [41]. Interventions addressing chronic effects were applied to two ABs per week over two weeks [24] to five ABs per week over ten weeks [46]. Overall, ABs consisted of aerobic or coordinative moderate PA (MPA) $[6,23,35,41,46,50]$, vigorous PA (VPA) $[6,44,45]$, or MVPA [19,41-43,47,49]. Two studies reported that the registered intensity was lower (i.e., light or light-to-moderate) than expected [24,51], and one did not report intensity-related data [48]. Regarding the type of PA, nine studies included cognitively engaging PA conditions (i.e., combined activities, games, dancing, or coordinative exercises) $[19,24,41-43,47-49,51]$, with two of them relating the PA to academic contents [24,49]; the other interventions included aerobic PA. Most of the interventions were delivered by the researchers $[6,19,23,24,35,41-43,45,51]$. Five of them were by the classroom teachers [6,47-50], and four of them also relied on videos to guide the ABs $[24,35,48,51]$.

\subsection{Methodological Quality}

The overall methodological quality of the intervention studies can be found in Table A2. Nine studies were assessed as having some concerns in their overall RoB 2 quality scale, and eight were assessed as high risk of bias. The score for the only study assessed by ROBINS-I was critical [48]. None of the studies achieved low risk of bias. Methodological assessment revealed issues on the quality of the bias in the information reported on the randomization process, the reporting of possible deviations from the intended interventions and the selection of the reported result.

\subsection{Active Breaks: Effects on Attention}

Due to the multi-component nature of attention, a wide variety of attention-related outcomes were reported among included studies (e.g., global attention; selective attention). Table A3 shows a synthesis of the outcomes according to the task and their scoring. Overall, results from the 11 parallel groups design studies (Table 2) showed that the effects of ABs on attention were mainly positive or non-significant; no negative effect of $\mathrm{ABs}$ was found. In addition, results from the five cross-over design studies (Table 3) showed similar results, with positive or no effects on attentional outcomes and without negative results. The results for each outcome are synthesized in the following sections.

\subsubsection{Effects on Accuracy}

Accuracy was only measured with the $\mathrm{d} 2$ test. Acute positive effects were found only in an aerobic MPA AB intervention on 9 to 11-year-old students [23]. No other acute $[19,45]$ nor chronic effects were found [48]. Meta-analyses could not be run since there were less than three studies per analysis. 
Table 2. Characteristics of the selected studies with a parallel-groups design.

\begin{tabular}{|c|c|c|c|c|c|c|}
\hline $\begin{array}{c}\text { Study } \\
\text { Risk of Bias }\end{array}$ & Design and AB Type & $\begin{array}{c}\text { Age (y.o.) Mean } \pm \text { SD } \\
\text { (Range) } \\
\text { Academic Level }\end{array}$ & $\begin{array}{l}\text { Sample Size }(n) \\
\quad \text { and Sex }\end{array}$ & $\begin{array}{l}\text { Attentional Outcomes } \\
\text { (Instrument) }\end{array}$ & Fitness Level & Results \\
\hline $\begin{array}{c}\text { Altenburg et al. (2016) } \\
\text { [35] } \\
\text { Some concerns }\end{array}$ & $\begin{array}{c}\text { RCT: two IG and one CG. } \\
\text { Acute. }\end{array}$ & $\begin{array}{l}\text { NR }(10-13) \\
\quad \text { NR }\end{array}$ & $\begin{array}{l}\text { All: } 52\left(29 \% 33 \sigma^{7}\right) \\
\text { IG1: } 17\left(5+12 \sigma^{7}\right) \\
\text { IG2: } 20\left(9 \div 11 \sigma^{7}\right) \\
\text { CG: } 19\left(12 \% 7 \sigma^{7}\right)\end{array}$ & $\begin{array}{l}\text { Selective attention (Sky } \\
\text { Search in TEA-Ch) }\end{array}$ & NR & $\begin{array}{l}\text { Children in IG2 (two ABs) } \\
\text { had better selective attention } \\
\text { than children in IG1 (one } \\
\text { AB) or CG. There was no } \\
\text { difference between IG1 } \\
\text { and CG. }\end{array}$ \\
\hline $\begin{array}{c}\text { Buchele et al. (2018) } \\
\text { [48] } \\
\text { Critical risk }\end{array}$ & $\begin{array}{l}\text { Non-randomized } \\
\text { quasi-experimental: two } \\
\text { IGs and one CG. } \\
\text { Chronic. }\end{array}$ & $\begin{array}{l}\text { NR }(\approx 10-11) \\
5 \text { th grade }\end{array}$ & $\begin{array}{l}\text { All: } 116\left(59+57 \sigma^{7}\right) \\
\text { IG1: } 31\left(14+17 \sigma^{7}\right) \\
\text { IG2: } 29\left(10 \% 19 \sigma^{7}\right) \\
\text { CG: } 56\left(35 \% 21 \sigma^{7}\right)\end{array}$ & $\begin{array}{c}\text { Accuracy }(\mathrm{d} 2)^{*} \\
\text { Concentration }(\mathrm{d} 2) \\
\text { Selective attention }(\mathrm{d} 2) \\
\text { Sustained attention }(\mathrm{d} 2) \\
*\end{array}$ & NR & $\begin{array}{l}\text { The IG1 increased all } \\
\text { attentional outcomes (except } \\
\text { accuracy) compared to the } \\
\text { CG and concentration and } \\
\text { sustained attention } \\
\text { compared to IG2 (no AB). } \\
\text { There were no differences } \\
\text { between IG2 and CG. }\end{array}$ \\
\hline $\begin{array}{l}\text { Egger et al. }(2018)[41] \\
\text { Some concerns }\end{array}$ & $\begin{array}{l}\text { RCT: three IGs and one } \\
\text { CG. } \\
\text { Acute. }\end{array}$ & $\begin{array}{l}\text { All: } 7.94 \pm 0.44(7-9) \\
\text { IG1: } 7.99 \pm 0.38 \\
\text { IG2: } 7.93 \pm 0.45 \\
\text { IG3: } 7.96 \pm 0.50 \\
\text { CG: } 7.90 \pm 0.44 \\
\text { 2nd grade }\end{array}$ & $\begin{array}{c}\text { All: } 216 \\
\left(\sim 1069110 \circ^{7}\right) \\
\text { IG1: } 59 \\
\text { IG2: } 53 \\
\text { IG3: } 50 \\
\text { CG: } 54\end{array}$ & $\begin{array}{l}\text { Inhibition reaction time } \\
(\mathrm{ms}) \text { (flanker task) } \\
\text { Shifting reaction time } \\
\text { (ms) (flanker task } \\
\text { additional block) }\end{array}$ & $\begin{array}{c}\text { Multistage 20m-SRT: IG1: } \\
304.58 \pm 123.18 . \text { IG2: } \\
284.27 \pm 141.16 . \text { IG3: } \\
306.43 \pm 144.23 . \text { CG: } \\
278.55 \pm 129.13\end{array}$ & $\begin{array}{c}\text { A significant, negative effect } \\
\text { was found for the CE factor } \\
\text { in shifting. No effects were } \\
\text { found for the PA factor or } \\
\text { the interaction between PA } \\
\text { and CE. }\end{array}$ \\
\hline $\begin{array}{l}\text { Jäger et al. (2014) [42] } \\
\text { Some concerns }\end{array}$ & $\begin{array}{c}\text { RCT: one IG and one CG. } \\
\text { Acute. }\end{array}$ & $\begin{array}{c}7.91 \pm 5.05 \text { (months) } \\
(6.83-8.92) \\
\text { 2nd grade }\end{array}$ & $\begin{array}{l}\text { All: } 104\left(57 \% 53 \sigma^{7}\right) \\
\text { IG: } 51\left(27+24 \sigma^{7}\right) \\
\text { CG: } 53\left(30+23 \sigma^{7}\right)\end{array}$ & $\begin{array}{l}\text { Inhibition reaction time } \\
(\mathrm{ms}) \text { (flanker task) } \\
\text { Shifting reaction time } \\
\text { (ms) (flanker task } \\
\text { additional block) }\end{array}$ & $\begin{array}{l}\text { Motor fitness: } 20 \mathrm{~m}-\mathrm{SRT} \\
\text { 20m sprint test and jump } \\
\text { side-to-side. }\end{array}$ & $\begin{array}{l}\text { The AB improved only } \\
\text { inhibition, and its effects } \\
\text { remained for less than } 40 \\
\text { min after the AB. The } \\
\text { improvements were } \\
\text { suggested to be independent } \\
\text { of the participants' } \\
\text { characteristics and stronger } \\
\text { among those with higher } \\
\text { increases in cortisol. }\end{array}$ \\
\hline
\end{tabular}


Table 2. Cont.

\begin{tabular}{|c|c|c|c|c|c|c|}
\hline $\begin{array}{c}\text { Study } \\
\text { Risk of Bias }\end{array}$ & Design and AB Type & $\begin{array}{c}\text { Age (y.o.) Mean } \pm \text { SD } \\
\text { (Range) } \\
\text { Academic Level }\end{array}$ & $\begin{array}{l}\text { Sample Size }(n) \\
\text { and Sex }\end{array}$ & $\begin{array}{l}\text { Attentional Outcomes } \\
\text { (Instrument) }\end{array}$ & Fitness Level & Results \\
\hline $\begin{array}{c}\text { Jäger et al. (2015) [43] } \\
\text { High risk }\end{array}$ & $\begin{array}{c}\text { RCT: three IGs and one } \\
\text { CG. } \\
\text { Acute. }\end{array}$ & $\begin{array}{c}11.29 \pm 6.53 \text { (months) } \\
(10.33-12.33) \\
\text { NR }\end{array}$ & $\begin{array}{l}\text { All: } 217\left(120 \% 97 \sigma^{7}\right) \\
\text { IG1: } 54\left(35+19 \sigma^{7}\right) \\
\text { IG2: } 62\left(28+34 \sigma^{7}\right) \\
\text { IG3: } 60\left(30 q 30 \sigma^{7}\right) \\
\text { CG: } 58\left(33+25 \sigma^{7}\right)\end{array}$ & $\begin{array}{l}\text { Inhibition reaction time } \\
\text { (ms) (flanker task) } \\
\text { Shifting reaction time } \\
\text { (ms) (flanker task } \\
\text { additional block) }\end{array}$ & $\begin{array}{c}\text { 18-mSRT: } \mathrm{VO}_{2} \max \\
\text { (ml/kg/min): Posttest: } \\
\text { IG1: } 46.77 \pm 6.73, \mathrm{IG} 2: \\
47.98 \pm 6.01, \mathrm{IG} 3: 46.77 \\
\pm 5.96), \mathrm{CG}: 47.58(6.12)\end{array}$ & $\begin{array}{l}\text { No effects of } \mathrm{AB} \text { (with and } \\
\text { without considering } \mathrm{CE} \text { ) } \\
\text { were found. Fitness did not } \\
\text { moderate the effects. }\end{array}$ \\
\hline $\begin{array}{c}\text { Niemann et al. (2013) } \\
\text { [44] } \\
\text { High risk }\end{array}$ & $\begin{array}{c}\text { RCT: one IG and one CG. } \\
\text { Acute. }\end{array}$ & $\begin{array}{l}9.69 \pm 0.44(9-10) \\
\text { IG: } 9.65 \pm 0.41 \\
\text { CG: } 9.74 \pm 0.48 \\
\text { 4th grade }\end{array}$ & $\begin{array}{c}\text { All: } 42 \\
\text { IG: } 27\left(13 \wp 14 \sigma^{\top}\right) \\
\text { CG: } 15\left(7 @ 8 \sigma^{7}\right)\end{array}$ & Concentration (d2) & NR & $\begin{array}{l}\text { The IG showed better } \\
\text { concentration than CG, } \\
\text { although both groups } \\
\text { improved from pre- to } \\
\text { post-test. There was an } \\
\text { interaction between group } \\
\text { (IG, CG) test (pre, post), and } \\
\text { PA level (high, low). }\end{array}$ \\
\hline $\begin{array}{c}\text { Ordóñez et al. (2019) } \\
\text { [46] } \\
\text { High risk }\end{array}$ & $\begin{array}{c}\text { CRT: one IG and one CG. } \\
\text { Chronic. }\end{array}$ & $\begin{array}{c}11.1(11-12) \\
\text { 6th grade (Spanish } \\
\text { Elementary Education) }\end{array}$ & $\begin{array}{l}\text { All: } 89 \\
\text { IG: } 45 \\
\text { CG: } 44\end{array}$ & $\begin{array}{c}\text { Concentration (FACES) } \\
\text { Selective attention } \\
\text { (FACES) }\end{array}$ & $\begin{array}{c}\text { ALPHA. } \\
\text { Lower-limb muscle } \\
\text { strength (meters): } \\
\text { Pretest: IG: } 1.36 \pm 0.21 ; \\
\text { CG: } 1.38 \pm 0.20 . \text { Posttest: } \\
\text { IG: } 1.42 \pm 0.21 \text {; CG: } 1.40 \\
\pm 0.21 \text {. Coordination (no. } \\
\text { jumps): Pretest: IG: } 28.33 \\
\pm \text { 6.89; CG: } 26.40 \pm 5.68 . \\
\text { Posttest: IG: } 30.87 \pm 5.68 ; \\
\text { CG: } 27.33 \pm 5.90 . \\
\text { Cardiorespiratory } \\
\text { capacity (min): Pretest: } \\
\text { IG: } 6.42 \pm 0.75 ; \text { CG:6.46 } \\
\pm 0.83 . \text { Posttest: IG: } 5.61 \\
\pm 0.68 ; \text { CG: } 6.20 \pm 0.75\end{array}$ & $\begin{array}{l}\text { Significant differences } \\
\text { between groups with higher } \\
\text { levels of attention in the IG. }\end{array}$ \\
\hline
\end{tabular}


Table 2. Cont.

\begin{tabular}{|c|c|c|c|c|c|c|}
\hline $\begin{array}{c}\text { Study } \\
\text { Risk of Bias }\end{array}$ & Design and AB Type & $\begin{array}{c}\text { Age (y.o.) Mean } \pm \text { SD } \\
\text { (Range) } \\
\text { Academic Level }\end{array}$ & $\begin{array}{l}\text { Sample Size }(n) \\
\quad \text { and Sex }\end{array}$ & $\begin{array}{l}\text { Attentional Outcomes } \\
\text { (Instrument) }\end{array}$ & Fitness Level & Results \\
\hline $\begin{array}{c}\text { Schmidt et al. (2016) } \\
\text { [19] } \\
\text { Some concerns }\end{array}$ & $\begin{array}{c}\text { RCT: three IGs and one } \\
\text { CG. } \\
\text { Acute. }\end{array}$ & $\begin{array}{c}11.77 \pm 0.41(11.01-12.98) \\
5 \text { th grade }\end{array}$ & $\begin{array}{l}\text { All: } 92\left(42+50 \sigma^{7}\right) \\
\text { IG1: } 25\left(\sim 12 \% 23 \sigma^{7}\right) \\
\text { IG2: } 22\left(10 \% 12 \sigma^{7}\right) \\
\text { IG3: } 25\left(11 \% 14 \sigma^{7}\right) \\
\text { CG: } 20\left(9+11 \sigma^{7}\right)\end{array}$ & $\begin{array}{c}\text { Accuracy }(\mathrm{d} 2)^{*} \\
\text { Concentration }(\mathrm{d} 2)\end{array}$ & NR & $\begin{array}{l}\text { No significant effects of } \mathrm{ABs} \\
\text { or their interactions with } \mathrm{CE} \\
\text { were found concerning } \\
\text { attention. However, high } \mathrm{CE} \\
\text { interventions had a positive } \\
\text { effect on focused attention, } \\
\text { and positive affect had a } \\
\text { mediational role between CE } \\
\text { factor, accuracy, and focused } \\
\text { attention, but not for PA. }\end{array}$ \\
\hline $\begin{array}{c}\text { Schmidt et al. (2019) } \\
\text { [24] } \\
\text { Some concerns }\end{array}$ & $\begin{array}{c}\text { CRT: two IGs and one } \\
\text { CG. } \\
\text { Chronic. }\end{array}$ & $\begin{array}{l}9.04 \pm 0.70 \\
\text { 3rd grade }\end{array}$ & $\begin{array}{c}\text { All: } 104\left(50954 \sigma^{7}\right) \\
\text { IG1: } 34 \\
\text { IG2: } 37 \\
\text { CG: } 33\end{array}$ & $\begin{array}{c}\text { Concentration (d2) } \\
\text { (measured after 3rd AB) }\end{array}$ & NR & $\begin{array}{l}\text { Focused attention did not } \\
\text { differ between the three } \\
\text { groups after controlling for } \\
\text { age, step counts, and } \\
\text { attention at pretest. }\end{array}$ \\
\hline $\begin{array}{l}\text { Van den Berg et al. } \\
\text { (2019) [47] } \\
\text { For most outcomes: } \\
\text { Some concernsFor d2: } \\
\text { High risk }\end{array}$ & $\begin{array}{l}\text { CRT: one IG and one CG. } \\
\text { Chronic. }\end{array}$ & $\begin{array}{c}\text { IG: } 10.8 \pm 0.6 \\
\text { CG: } 10.9 \pm 0.7 \\
\quad(9-12) \\
5 \text { th-6th grade }\end{array}$ & $\begin{array}{l}\text { All: } 510 \text { ( } 448 \text { to } 467 \text {, } \\
\text { depending on the } \\
\text { outcome). } \\
\text { IG: } 100\left(46954 \sigma^{7}\right) \\
\text { CG: } 100\left(479530^{7}\right)\end{array}$ & $\begin{array}{l}\text { Alerting reaction time } \\
(\mathrm{ms}) \text { and accuracy }(\%) \\
(\text { ANT) } \\
{ }^{*} \text { Concentration }(\mathrm{d} 2) \\
\text { Inhibition reaction time } \\
(\mathrm{ms}) \text { and accuracy }(\%) \\
(\text { ANT a and Stroop Color } \\
\left.\text { Word Task }{ }^{*}\right) \\
\text { Orienting reaction time } \\
(\mathrm{ms}) \text { and accuracy }(\%) \\
(\mathrm{ANT}){ }^{*}\end{array}$ & $\begin{array}{l}\text { 18-mSRT: } \mathrm{VO}_{2} \max \\
\text { (ml/kg/min): Pretest: } \\
\text { IG: } 48.1 \pm 5.0 \text {; CG: } 48.0 \\
\pm \text { 5.0. Posttest: IG: } 48.9 \\
\pm 0.2 ; \text { CG: } 48.8 \pm 0.2\end{array}$ & $\begin{array}{l}\text { No intervention effects were } \\
\text { detected on any outcome } \\
\text { after controlling for pretest } \\
\text { score, age, arithmetic } \\
\text { performance, class, and } \\
\text { school. } \\
\text { The IG spent more time in } \\
\text { MVPA, but their fitness } \\
\text { levels were similar to } \\
\text { students in the CG. }\end{array}$ \\
\hline
\end{tabular}

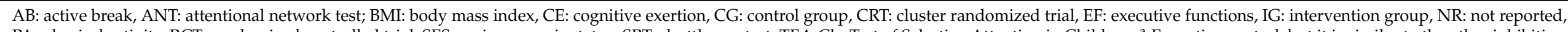

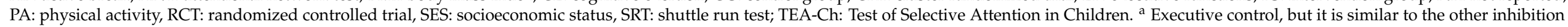
tasks. Therefore, results were treated as inhibition. * Not included in the meta-analysis. 
Table 3. Characteristics of the selected studies with a cross-over design *.

\begin{tabular}{|c|c|c|c|c|c|c|}
\hline $\begin{array}{c}\text { Study } \\
\text { Risk of Bias }\end{array}$ & Design and Type of $A B$ & $\begin{array}{c}\text { Age (y.o.) Mean } \pm \text { SD } \\
\text { (Range) } \\
\text { Academic Level }\end{array}$ & Sample Size $(n) /$ Sex & $\begin{array}{c}\text { Outcomes } \\
\text { (Instruments/Tasks) }\end{array}$ & Fitness Level & Results \\
\hline $\begin{array}{l}\text { Hill et al. (2010) [50] } \\
\text { Some concerns }\end{array}$ & $\begin{array}{c}\text { CRT counterbalanced } \\
\text { with two conditions. } \\
\text { Acute. }\end{array}$ & $\begin{array}{c}\text { NR } \\
(8-11) \\
\text { 4th-7th grade (Scottish) }\end{array}$ & $\begin{array}{c}\text { All: } 1224 \text { (1074 } \\
\text { completed three or more } \\
\text { of the tests on both } \\
\text { weeks) }\end{array}$ & $\begin{array}{l}\text { Global attention: overall } \\
\text { performance of different } \\
\text { executive functions tests) }\end{array}$ & - & $\begin{array}{c}\text { AB improved attention } \\
\text { only among participants } \\
\text { who received the } \\
\text { intervention in the } \\
\text { second period. } \\
\text { Improvements were } \\
\text { moderated by test and } \\
\text { age. }\end{array}$ \\
\hline $\begin{array}{c}\text { Janssen et al. (2014) [6] } \\
\text { High risk }\end{array}$ & $\begin{array}{l}\text { Single group. Three } \\
\text { randomized conditions } \\
\text { at the group level. } \\
\text { Acute. }\end{array}$ & $\begin{array}{l}10.4 \pm 0.59 \\
(10-11) \\
5 \text { th grade }\end{array}$ & All: $123\left(619620^{7}\right)$ & $\begin{array}{l}\text { Selective attention (Sky } \\
\text { Search in TEA-Ch) }\end{array}$ & $\begin{array}{l}\text { 20-mSRT (dichotomized } \\
\text { into high or low) }\end{array}$ & $\begin{array}{c}\text { Attention was } \\
\text { significantly better in all } \\
\text { the conditions than in } \\
\text { the 'no break' condition } \\
\text { Attention scores were } \\
\text { best after the MPA AB. } \\
\text { Attention after VPA } \\
\text { breaks was better than } \\
\text { after no break but was } \\
\text { no different than after } \\
\text { the passive break. No } \\
\text { moderation effect of } \\
\text { fitness was detected. }\end{array}$ \\
\hline $\begin{array}{c}\text { Ma et al. (2015) [23] } \\
\text { High risk }\end{array}$ & $\begin{array}{l}\text { Single group (divided). } \\
\text { Two randomized } \\
\text { conditions at the group } \\
\text { level. } \\
\text { Acute (mean of several } \\
\text { acutes). }\end{array}$ & $\begin{array}{c}\text { NR } \\
(9-11) \\
3-5 \text { th grade }\end{array}$ & 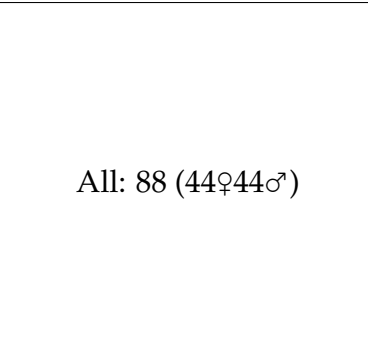 & $\begin{array}{c}\text { Accuracy }(\mathrm{d} 2) \\
\text { Concentration }(\mathrm{d} 2) \\
\text { Selective attention }(\mathrm{d} 2)\end{array}$ & - & $\begin{array}{c}\text { Better processing speed } \\
\text { scores were reported } \\
\text { after no AB. Accuracy } \\
\text { improved after the AB. } \\
\text { No effects on selective } \\
\text { attention were observed } \\
\text { following the AB, } \\
\text { although accuracy } \\
\text { improved. }\end{array}$ \\
\hline
\end{tabular}


Table 3. Cont.

\begin{tabular}{|c|c|c|c|c|c|c|}
\hline $\begin{array}{c}\text { Study } \\
\text { Risk of Bias }\end{array}$ & Design and Type of $A B$ & $\begin{array}{c}\text { Age (y.o.) Mean } \pm \text { SD } \\
\text { (Range) } \\
\text { Academic Level }\end{array}$ & Sample Size $(n) /$ Sex & $\begin{array}{c}\text { Outcomes } \\
\text { (Instruments/Tasks) }\end{array}$ & Fitness Level & Results \\
\hline $\begin{array}{c}\text { van den Berg et al. (2016) } \\
\text { [51] } \\
\text { Some concerns }\end{array}$ & $\begin{array}{c}\text { CRT counterbalanced } \\
\text { with two conditions in } \\
\text { three different groups. } \\
\text { Acute. }\end{array}$ & $\begin{array}{c}11.7 \pm 0.7 \\
(10-13) \\
5 \text { th-6th grade }\end{array}$ & $\begin{array}{l}\text { All: } 184\left(46+54 \sigma^{7}\right) \\
\text { IC1: } 66\left(47+53 \sigma^{7}\right) \\
\text { IC2: } 71\left(44+56 \sigma^{7}\right) \\
\text { IC3: } 47\left(49+51 \sigma^{7}\right)\end{array}$ & Concentration (d2) & - & $\begin{array}{c}\text { No effects of ABs } \\
\text { (LMPA) on attention } \\
\text { were reported nor were } \\
\text { differential effects of } \\
\text { exercise type, after } \\
\text { controlling for age and } \\
\text { session order. Scores for } \\
\text { both conditions } \\
\text { improved from day } 1 \text { to } \\
\text { day } 2 \text {. }\end{array}$ \\
\hline $\begin{array}{l}\text { Wilson et al. (2016) [49] } \\
\text { Some concerns }\end{array}$ & $\begin{array}{l}\text { Single group. Two } \\
\text { randomized conditions } \\
\text { at the group level. } \\
\text { Acute (mean of several } \\
\text { acutes). }\end{array}$ & $\begin{array}{c}11.2 \pm 0.6 \\
(\approx 10-12) \\
5 \text { th-6th grade }\end{array}$ & All: $580^{\pi}$ & $\begin{array}{c}\text { Vigilance Mean Reaction } \\
\text { Time (ms) and lapses (\%) } \\
(\text { PVT) }\end{array}$ & - & $\begin{array}{l}\text { There were no } \\
\text { significant differences } \\
\text { between the } A B \text { and } \\
\text { no-AB conditions. }\end{array}$ \\
\hline
\end{tabular}

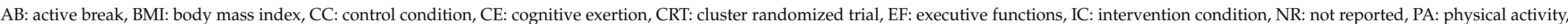

PVT: psychomotor vigilance task; RCT: randomized controlled trial, SES: socioeconomic status, SRT: shuttle run test; TEA-Ch: Test of Selective Attention in Children. * Not included in the meta-analysis. 
Table 4. Protocols of interventions.

\begin{tabular}{|c|c|c|c|c|c|c|c|c|c|}
\hline Study ID & Type of AB & CG/CC Activity & $\begin{array}{c}\text { AB } \\
\text { Duration } \\
\text { (Min) }\end{array}$ & $\begin{array}{l}\text { Duration and } \\
\text { Weekly/Daily } \\
\text { Freq. }\end{array}$ & Time of AB & $\begin{array}{l}\text { Intensity and } \\
\text { Type of PA }\end{array}$ & Responsible & $\begin{array}{l}\text { Timing of } \\
\text { Pre-Test and } \\
\text { Post-Test }\end{array}$ & Fidelity \\
\hline $\begin{array}{l}\text { Altenburg et al. } \\
\text { (2016) [35] }\end{array}$ & $\begin{array}{l}\text { IG1: One } 20-\min \text { AB. } \\
\text { IG2: Two } 20-\text { min PA } \\
\text { bouts. }\end{array}$ & $\begin{array}{l}\text { CG: No PA. } \\
\text { Sitting all } \\
\text { morning } \\
\text { working on } \\
\text { simulated } \\
\text { school tasks. }\end{array}$ & 20 & $\begin{array}{c}\text { NA } \\
\text { IG1: } 1 \text { t-d; IG2: } 2 \\
\text { t-d }\end{array}$ & $\begin{array}{l}\text { IG1: after } 90 \\
\text { min of sitting. } \\
\text { IG2: one } \mathrm{AB} \text { at } \\
\text { the start and } \\
\text { another after } 90 \\
\text { min of sitting. }\end{array}$ & $\begin{array}{l}\text { MPA. Aerobic. } \\
\text { No AC. }\end{array}$ & $\begin{array}{l}\text { Supervising } \\
\text { research staff } \\
\text { with videos }\end{array}$ & $\begin{array}{c}\text { Pre: At baseline } \\
\text { (T0). } \\
\text { Post: After } 20 \\
\text { min of school, } \\
\text { after } 130 \text { min; } \\
\text { and after } 220 \\
\text { min. }\end{array}$ & HR monitor \\
\hline $\begin{array}{c}\text { Buchele et al. } \\
\text { (2018) [48] }\end{array}$ & $\begin{array}{l}\text { IG1 “Coordinated } \\
\text { bilateral PA". } \\
\text { IG2 * "Fitbit Only": } \\
\text { Participants wore HR } \\
\text { monitors on weekly } \\
\text { days with no addition } \\
\text { instructions. }\end{array}$ & $\begin{array}{c}\text { CG: Usually } \\
\text { scheduled } \\
\text { school academic } \\
\text { instruction } \\
\text { periods while } \\
\text { wearing plastic } \\
\text { wristbands. }\end{array}$ & 6 & $\begin{array}{c}4 \text { weeks } \\
5 \mathrm{~d}-\mathrm{w} / 1 \mathrm{t}-\mathrm{d}\end{array}$ & $\begin{array}{c}\text { After } 20 \text { min of } \\
\text { sedentary } \\
\text { behavior. }\end{array}$ & $\begin{array}{c}\text { NA } \\
\text { IG1 Coordina- } \\
\text { tion.IG2: no PA. } \\
\text { No AC. }\end{array}$ & $\begin{array}{l}\text { Teachers with } \\
\text { videos }\end{array}$ & $\begin{array}{c}\text { Pre: The } \\
\text { previous week } \\
\text { the intervention. } \\
\text { Post: The week } \\
\text { after the } \\
\text { intervention. }\end{array}$ & NR \\
\hline $\begin{array}{l}\text { Egger et al. } \\
\text { (2018) [41] }\end{array}$ & $\begin{array}{l}\text { IG1 “Combo: high CE } \\
\text { + high PA": Running } \\
\text { while listening to a } \\
\text { song with keywords } \\
\text { to perform specific } \\
\text { actions and inhibit } \\
\text { others. } \\
\text { IG2 * "Cognition: } \\
\text { high CE + low PA": } \\
\text { Sitting while listening } \\
\text { and reacting to a song. } \\
\text { IG3 “Aerobic: low CE } \\
\text { + high PA": Running } \\
\text { while listening to a } \\
\text { song, but without } \\
\text { changing the actions } \\
\text { performed. }\end{array}$ & $\begin{array}{c}\text { CG “Low CE + } \\
\text { low PA": } \\
\text { Participants sat } \\
\text { comfortably in a } \\
\text { circle and } \\
\text { listened to an } \\
\text { age-appropriate } \\
\text { audio-book for } \\
20 \text { min. }\end{array}$ & $\approx 25^{\mathrm{a}}$ & NA & $\begin{array}{c}\text { Morning } \\
\text { (9:25-9:50 a.m.) }\end{array}$ & $\begin{array}{l}\text { MVPA. } \\
\text { IG1: Cognitive. } \\
\text { IG2: no PA. } \\
\text { IG3: Aerobic.No } \\
\text { AC. }\end{array}$ & Researcher & $\begin{array}{c}\text { Pre: Before the } \\
\text { AB (9:05-9:25 } \\
\text { a.m.). } \\
\text { Post: } \\
\text { Immediately } \\
\text { after the AB } \\
\text { (9:50-10:10 } \\
\text { a.m.). }\end{array}$ & $\begin{array}{c}\text { HR monitors } \\
\text { and Borg RPE } \\
\text { scale. } \\
\text { Perceived CE } \\
\text { was also } \\
\text { assessed }\end{array}$ \\
\hline
\end{tabular}


Table 4. Cont.

\begin{tabular}{|c|c|c|c|c|c|c|c|c|c|}
\hline Study ID & Type of AB & CG/CC Activity & $\begin{array}{c}\mathrm{AB} \\
\text { Duration } \\
\text { (Min) }\end{array}$ & $\begin{array}{c}\text { Duration and } \\
\text { Weekly/Daily } \\
\text { Freq. }\end{array}$ & Time of AB & $\begin{array}{c}\text { Intensity and } \\
\text { Type of PA }\end{array}$ & Responsible & $\begin{array}{l}\text { Timing of } \\
\text { Pre-Test and } \\
\text { Post-Test }\end{array}$ & Fidelity \\
\hline $\begin{array}{l}\text { Hill et al. (2010) } \\
\text { [50] }\end{array}$ & $\begin{array}{l}\text { IC: Stretching and } \\
\text { aerobic PA (e.g., } \\
\text { running on the spot, } \\
\text { hopping sequences to } \\
\text { music). }\end{array}$ & $\begin{array}{l}\text { CC: Normal } \\
\text { curriculum } \\
\text { plan. }\end{array}$ & $10-15$ & $\begin{array}{c}2 \text { weeks } \\
5 \mathrm{~d}-\mathrm{w} / 1 \mathrm{t}-\mathrm{d}\end{array}$ & $\begin{array}{c}\approx 30 \text { min after } \\
\text { lunch. }\end{array}$ & $\begin{array}{l}\text { MPA. Aerobic. } \\
\text { No AC. }\end{array}$ & Trained teachers & $\begin{array}{c}\text { Pre: NR. } \\
\text { Post: At the end } \\
\text { of the school } \\
\text { day. }\end{array}$ & $\begin{array}{l}\text { Teachers' } \\
\text { control }\end{array}$ \\
\hline $\begin{array}{l}\text { Jäger et al. } \\
\text { (2014) [42] }\end{array}$ & $\begin{array}{c}\text { IG "EF-specific } \\
\text { cognitive engaging } \\
\text { PA": Warm-up with a } \text { song, playing tag, and } \\
\text { balancing on various } \\
\text { objects. }\end{array}$ & $\begin{array}{c}\text { CG: } 15 \text { min } \\
\text { seated on a mat } \\
\text { while listening } \\
\text { to an } \\
\text { age-appropriate } \\
\text { story. The last } 5 \\
\text { min were spent } \\
\text { answering easy } \\
\text { questions. }\end{array}$ & $\approx 20$ & NA & 10:00-10:20 a.m. & $\begin{array}{l}\text { MVPA. } \\
\text { Cognitive. } \\
\text { No AC. }\end{array}$ & Researcher & $\begin{array}{l}\text { Pre: Prior the } \\
\text { intervention. } \\
\text { Post: Just after } \\
\text { the AB. } \\
\text { Follow-up } 40 \\
\text { min after. }\end{array}$ & HR monitors \\
\hline $\begin{array}{l}\text { Jäger et al. } \\
\text { (2015) [43] }\end{array}$ & $\begin{array}{l}\text { IG1 “Physical games: } \\
\text { PA + CE": three } \\
\text { different cooperative } \\
\text { and competitive PA } \\
\text { games involving EF. } \\
\text { IG2 “Aerobic } \\
\text { exercise": Short tasks } \\
\text { and games with } \\
\text { different forms of } \\
\text { running. } \\
\text { IG3 * "Cognitive } \\
\text { games: Sedentary + } \\
\text { CE": card game. }\end{array}$ & $\begin{array}{l}\text { CG: Sedentary } \\
\text { without CE: } \\
\text { Participants sat } \\
\text { comfortably on } \\
\text { a mat and } \\
\text { listened to an } \\
\text { age-appropriate } \\
\text { story. }\end{array}$ & 20 & NA & NR & $\begin{array}{l}\text { MVPA. } \\
\text { IG1: Cognitive } \\
\text { IG2: Aerobic. } \\
\text { IG3: no PA. } \\
\text { No AC. }\end{array}$ & Researcher & $\begin{array}{l}\text { Pre: Just before } \\
\text { the intervention. } \\
\text { Post: } \\
\text { Immediately } \\
\text { after the } \\
\text { intervention. }\end{array}$ & HR monitors \\
\hline
\end{tabular}


Table 4. Cont

\begin{tabular}{|c|c|c|c|c|c|c|c|c|c|}
\hline Study ID & Type of AB & CG/CC Activity & $\begin{array}{c}\text { AB } \\
\text { Duration } \\
\text { (Min) }\end{array}$ & $\begin{array}{c}\text { Duration and } \\
\text { Weekly/Daily } \\
\text { Freq. }\end{array}$ & Time of AB & $\begin{array}{l}\text { Intensity and } \\
\text { Type of PA }\end{array}$ & Responsible & $\begin{array}{l}\text { Timing of } \\
\text { Pre-Test and } \\
\text { Post-Test }\end{array}$ & Fidelity \\
\hline $\begin{array}{c}\text { Janssen et al. } \\
\text { (2014) [6] }\end{array}$ & $\begin{array}{l}\text { IC1 "MPA-AB": } \\
\text { Walking to and from } \\
\text { the PE classroom, } \\
\text { jogging, and passing } \\
\text { and dribbling a ball. } \\
\text { IC2 “VPA-AB": } \\
\text { Running to and from } \\
\text { the PE classroom, } \\
\text { running, jumping, } \\
\text { and rope skipping. }\end{array}$ & $\begin{array}{c}\text { CC1: No break. } \\
\text { Participants } \\
\text { were not } \\
\text { allowed to ask } \\
\text { the teacher for } \\
\text { help or go to the } \\
\text { toilet. } \\
\text { CC2: Passive } \\
\text { break. The } \\
\text { teacher read a } \\
\text { story to the } \\
\text { participants. }\end{array}$ & 15 & NA & $\begin{array}{l}\text { After an hour of } \\
\text { regular } \\
\text { cognitive tasks } \\
\text { (9:30-10:00 a.m.) }\end{array}$ & $\begin{array}{l}\text { IC1: MPA: } \\
\text { Aerobic. } \\
\text { IC2: VPA: } \\
\text { Aerobic. } \\
\text { No AC. }\end{array}$ & $\begin{array}{l}\text { Two researchers } \\
\text { and the } \\
\text { classroom } \\
\text { teacher }\end{array}$ & $\begin{array}{l}\text { Pre: Before and } \\
\text { after each } \\
\text { experimental } \\
\text { break in the } \\
\text { classroom. } \\
\text { Post: After each } \\
\text { experimental } \\
\text { break in the } \\
\text { classroom. }\end{array}$ & Accelerometry \\
\hline $\begin{array}{c}\text { Ma et al. (2015) } \\
\text { [23] }\end{array}$ & $\begin{array}{l}\text { IC “FUNtervals": } \\
\text { eight } 20 \text { s periods of } \\
\text { VPA (i.e., squats, } \\
\text { jumping jacks, scissor } \\
\text { kicks, jumping on the } \\
\text { spot) separated by } \\
\text { 10-s rest periods. }\end{array}$ & $\begin{array}{c}\text { CC: } 10-\text { min } \\
\text { lecture } \\
\text { separated from } \\
\text { recess by at least } \\
20 \text { min of } \\
\text { normal } \\
\text { classroom } \\
\text { instruction. }\end{array}$ & $10^{\mathrm{a}}(4)$ & $\begin{array}{l}3 \text { weeks. } \\
\text { On two separate } \\
\text { days in random. }\end{array}$ & $\begin{array}{l}\text { After at least } 20 \\
\text { min of normal } \\
\text { classroom } \\
\text { instruction } \\
\text { following the } \\
\text { recess. }\end{array}$ & $\begin{array}{l}\text { MPA. Aerobic } \\
\text { No AC. }\end{array}$ & Researcher & $\begin{array}{l}\text { Pre: In week 1, } \\
\text { familiarization. } \\
\text { Post: after } \\
\text { 10-min } \\
\text { researcher- } \\
\text { delivered } \\
\text { lecture } \\
\text { following AB. }\end{array}$ & $\begin{array}{l}\text { Teachers' } \\
\text { control }\end{array}$ \\
\hline $\begin{array}{l}\text { Niemann et al. } \\
\text { (2013) [44] }\end{array}$ & $\begin{array}{l}\text { IG: Running on a } 400 \\
\text { m track. Participants } \\
\text { were not allowed to } \\
\text { talk to each other and } \\
\text { remained silent. }\end{array}$ & $\begin{array}{c}\text { CG: Participants } \\
\text { performed } \\
\text { sedentary } \\
\text { behavior while } \\
\text { watching } \\
\text { non-arousing } \\
\text { scenes. } \\
\text { Participants } \\
\text { were not } \\
\text { allowed to talk } \\
\text { to each other } \\
\text { and remained } \\
\text { silent. }\end{array}$ & 12 & NA & After 11:30 a.m. & $\begin{array}{l}\text { VPA. Aerobic. } \\
\text { No AC. }\end{array}$ & NR & $\begin{array}{l}\text { Pre: After four } \\
\text { normal school } \\
\text { lessons just } \\
\text { before AB. } \\
\text { Post: } 5 \text { min after } \\
\text { AB. }\end{array}$ & $\begin{array}{c}\text { Control of } \\
\text { prior PA in } \\
\text { interventions } \\
\text { days }\end{array}$ \\
\hline
\end{tabular}


Table 4. Cont.

\begin{tabular}{|c|c|c|c|c|c|c|c|c|c|}
\hline Study ID & Type of AB & CG/CC Activity & $\begin{array}{c}\text { AB } \\
\text { Duration } \\
\text { (Min) }\end{array}$ & $\begin{array}{c}\text { Duration and } \\
\text { Weekly/Daily } \\
\text { Freq. }\end{array}$ & Time of AB & $\begin{array}{c}\text { Intensity and } \\
\text { Type of PA }\end{array}$ & Responsible & $\begin{array}{l}\text { Timing of } \\
\text { Pre-Test and } \\
\text { Post-Test }\end{array}$ & Fidelity \\
\hline $\begin{array}{l}\text { Ordóñez et al. } \\
\text { (2019) [46] }\end{array}$ & $\begin{array}{l}\text { IG: The first two } \\
\text { weeks: running a } \\
\text { 250-m circuit inside } \\
\text { the school; the next } \\
\text { four weeks: } 500 \mathrm{~m} \text {; } \\
\text { and in the last four } \\
\text { weeks: } 750 \mathrm{~m} \text {. }\end{array}$ & CG: No AB. & NA & $\begin{array}{c}10 \text { weeks } \\
5 \mathrm{~d}-\mathrm{w} / 1 \mathrm{t}-\mathrm{d}\end{array}$ & $\begin{array}{l}\text { Between the 2nd } \\
\text { and 3rd lesson } \\
\text { in the morning. }\end{array}$ & $\begin{array}{l}\text { MPA. Aerobic. } \\
\text { No AC. }\end{array}$ & NR & $\begin{array}{l}\text { Pre: At the same } \\
\text { time with both } \\
\text { groups, just } \\
\text { before AB. } \\
\text { Post: NR. }\end{array}$ & $\begin{array}{l}\text { Prior familiar- } \\
\text { ization for } \\
\text { maintaining } \\
\text { MPA }\end{array}$ \\
\hline $\begin{array}{l}\text { Schmidt et al. } \\
\text { (2016) [19] }\end{array}$ & $\begin{array}{l}\text { IG1 “Combo: high } \\
\text { CE + high PA": } \\
\text { PA-based activity of } \\
\text { adding numbers. } \\
\text { IG2 “Cognition: high } \\
\text { CE + low PA": A } \\
\text { paper } \\
\text {-and-pencil } \\
\text { trail-making test. } \\
\text { IG3 “Aerobic: low CE } \\
\text { + high PA": Running } \\
\text { at different speeds. }\end{array}$ & $\begin{array}{l}\text { CG "sedentary + } \\
\text { low CE": } \\
\text { Students } \\
\text { remained at } \\
\text { their desks in } \\
\text { the classroom } \\
\text { and listened to } \\
\text { an } \\
\text { age-appropriate } \\
\text { story for } 10 \text { min } \\
\text { to relax and } \\
\text { enjoy. }\end{array}$ & 10 & NA & $\begin{array}{l}\text { After } 20 \text { min of } \\
\text { German } \\
\text { language class } \\
(11: 15-11: 30 \\
\text { a.m. })\end{array}$ & $\begin{array}{l}\text { MVPA. } \\
\text { IG1: Cognitive } \\
\text { IG2: no PA. } \\
\text { IG3: Aerobic. } \\
\text { No AC. }\end{array}$ & Researchers & $\begin{array}{l}\text { Pre: Before AB } \\
\text { (10:45-10:55 } \\
\text { a.m.). } \\
\text { Post: } \\
\text { Immediately } \\
\text { after AB } \\
\text { (11:30-11:40 } \\
\text { a.m.). }\end{array}$ & $\begin{array}{c}\text { HR monitors, } \\
\text { Borg scale, } \\
\text { and } \\
\text { self-perceived } \\
\text { CE }\end{array}$ \\
\hline $\begin{array}{l}\text { Schmidt et al. } \\
\text { (2019) [24] }\end{array}$ & $\begin{array}{l}\text { IG1 “Embodied } \\
\text { learning condition": } \\
\text { PA-based learning } \\
\text { French vocabulary. } \\
\text { IG2 "PA condition": } \\
\text { Movements at the } \\
\text { same intensity } \\
\text { without academic } \\
\text { content. }\end{array}$ & $\begin{array}{l}\text { CG: Sedentary } \\
\text { teaching style } \\
\text { (words were } \\
\text { repeated equally } \\
\text { as under other } \\
\text { conditions). }\end{array}$ & 10 & $\begin{array}{c}2 \text { weeks } \\
2 \mathrm{~d}-\mathrm{w} / 1 \mathrm{t}-\mathrm{d}\end{array}$ & $\begin{array}{l}\text { 10:00 am-12:00 } \\
\text { p.m. }\end{array}$ & $\begin{array}{c}\text { LPA } \\
\text { IG1: Cognitive } \\
\text { IG2: Aerobic. } \\
\text { AC: IG1: } \\
\text { earning animals } \\
\text { in French; IG2: } \\
\text { No. }\end{array}$ & $\begin{array}{c}\text { Trained research } \\
\text { student with a } \\
\text { video }\end{array}$ & $\begin{array}{l}\text { Pre: Before the } \\
\text { beginning of the } \\
\text { first learning } \\
\text { session. } \\
\text { Post: } \\
\text { Immediately } \\
\text { after the third } \\
\text { learning session. }\end{array}$ & Accelerometry \\
\hline
\end{tabular}


Table 4. Cont.

\begin{tabular}{|c|c|c|c|c|c|c|c|c|c|}
\hline Study ID & Type of AB & CG/CC Activity & $\begin{array}{c}\text { AB } \\
\text { Duration } \\
\text { (Min) }\end{array}$ & $\begin{array}{c}\text { Duration and } \\
\text { Weekly/Daily } \\
\text { Freq. }\end{array}$ & Time of AB & $\begin{array}{l}\text { Intensity and } \\
\text { Type of PA }\end{array}$ & Responsible & $\begin{array}{l}\text { Timing of } \\
\text { Pre-Test and } \\
\text { Post-Test }\end{array}$ & Fidelity \\
\hline $\begin{array}{c}\text { Tine et al. (2012) } \\
\text { [45] }\end{array}$ & $\begin{array}{l}\text { IG: Running around } \\
\text { an indoor track. }\end{array}$ & $\begin{array}{l}\text { CG: Students } \\
\text { remained seated } \\
\text { and viewed a } \\
\text { 12-min film } \\
\text { video. }\end{array}$ & 12 & NA & $\begin{array}{l}2 \text { sessions on } \\
\text { separate days } \\
\text { during usual } \\
\text { gym classes. }\end{array}$ & $\begin{array}{l}\text { VPA. Aerobic. } \\
\text { No AC. }\end{array}$ & Researchers & $\begin{array}{c}\text { Pre: Just before } \\
\text { AB. } \\
\text { Post: One } \\
\text { minute after AB. }\end{array}$ & HR monitors \\
\hline $\begin{array}{l}\text { van den Berg } \\
\text { et al. (2016) [51] }\end{array}$ & $\begin{array}{l}\text { IC1 "Aerobic": Easy } \\
\text { and repetitive } \\
\text { movements. } \\
\text { IC2 “Coordination": } \\
\text { Complex movements } \\
\text { stressing coordinative } \\
\text { skills. } \\
\text { IC3 "Strength": } \\
\text { Dynamic and static } \\
\text { body-weight exercises } \\
\text { adjusted to the age. }\end{array}$ & $\begin{array}{l}\text { CC: } 12 \text { min of } \\
\text { sitting and } \\
\text { listening to an } \\
\text { educational } \\
\text { lesson about } \\
\text { exercise and } \\
\text { movement. }\end{array}$ & 12 & NA & 8:30-10:00 a.m. & $\begin{array}{l}\text { LMPA (target: } \\
\text { MVPA). } \\
\text { IC1: Aerobic. } \\
\text { IC2: } \\
\text { Coordination. } \\
\text { IC3: Strength. } \\
\text { No AC. }\end{array}$ & $\begin{array}{c}\text { Researcher, } \\
\text { three research } \\
\text { assistants, and } \\
\text { standardized } \\
\text { movie }\end{array}$ & $\begin{array}{c}\text { Pre: Just before } \\
\text { AB. } \\
\text { Post: } \\
\text { Immediately } \\
\text { after AB. }\end{array}$ & $\begin{array}{l}\text { HR monitor, } \\
\text { familiariza- } \\
\text { tion and } \\
\text { control of } \\
\text { previous } \\
\text { bedtime, } \\
\text { breakfast, and } \\
\text { transport to } \\
\text { school }\end{array}$ \\
\hline $\begin{array}{l}\text { van den Berg } \\
\text { et al. (2019) [47] }\end{array}$ & $\begin{array}{l}\text { IG: Following three } \\
\text { "Just Dance" videos. }\end{array}$ & $\begin{array}{c}\text { CG: Nine } 10-15 \\
\text { min educational } \\
\text { lessons once a } \\
\text { week. }\end{array}$ & 10 & $\begin{array}{c}9 \text { weeks } \\
5 \mathrm{~d}-\mathrm{w} / 1 \mathrm{t}-\mathrm{d}\end{array}$ & NR & $\begin{array}{l}\text { MVPA. } \\
\text { Dancing. } \\
\text { No AC. }\end{array}$ & Teachers & $\begin{array}{l}\text { Pre: The week } \\
\text { before the } \\
\text { intervention } \\
\text { started. } \\
\text { Post: The } \\
\text { following week } \\
\text { after the } \\
\text { intervention. }\end{array}$ & $\begin{array}{c}\text { Accelerometry } \\
\text { Teachers' } \\
\text { control. }\end{array}$ \\
\hline $\begin{array}{l}\text { Wilson et al. } \\
\text { (2016) [49] }\end{array}$ & $\begin{array}{c}\text { IC "Active Lesson } \\
\text { Breaks" outside the } \\
\text { regular classroom, } \\
\text { including tag/chasing } \\
\text { games, or } \\
\text { invasion-type games. }\end{array}$ & $\begin{array}{c}\text { CC: Passive } \\
\text { lesson break: } \\
\text { Participants } \\
\text { spent } 10 \text { min } \\
\text { sitting outside } \\
\text { their classroom } \\
\text { reading. }\end{array}$ & 10 & $\begin{array}{c}8 \text { weeks each }+2 \\
\text { weeks of } \\
\text { washout. } \\
3 \mathrm{~d}-\mathrm{w} / 1 \mathrm{t}-\mathrm{d}\end{array}$ & - & $\begin{array}{l}\text { MVPA. } \\
\text { Cognitive. } \\
\text { AC: based on } \\
\text { Take10! and } \\
\text { Energizers, or } \\
\text { Texas I CAN. }\end{array}$ & Trained teacher & $\begin{array}{l}\text { Pre: } 5 \text { min } \\
\text { before AB. } \\
\text { Post: } \\
\text { Immediately } \\
\text { after AB. }\end{array}$ & Accelerometry \\
\hline
\end{tabular}

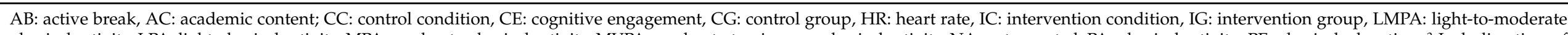

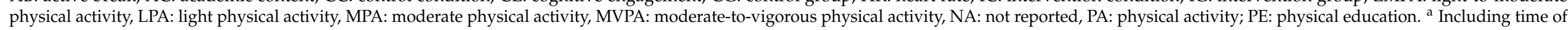
preparation. * Not AB: Intervention group or condition that did not include ABs. Groups and conditions are reported in bold letters to improve legibility. 


\subsubsection{Effects on Inhibition}

Inhibition was measured in four studies through the flanker task, the Stroop task, and the ANT tests. Only one study found acute favorable effects after a 20-min cognitively engaged $A B$ intervention [42]. The remaining studies on acute [41,43] or chronic [47] effects found no significant results.

Regarding the meta-analysis, four studies provided data for inhibition (i.e., reaction time results from flanker and ANT) involving six experimental and four control groups (pooled $n=900$ ). There was a trivial effect of $\mathrm{AB}$ on inhibition ( $\mathrm{ES}=0.08 ; 95 \% \mathrm{CI}=-0.07$ to $0.23 ; p=0.293 ; I^{2}=12.0 \%$; Egger's test $p=0.576$; relative weight of each group: 9.9 to $44.7 \%$; Figure 2). After study-by-study and group-by-group sensitivity analyses, no significant changes in results were noted (i.e., $p$-value remained at $>0.05$, mean ES $=0.02$ to 0.14 ). No significant sub-group differences $(p=0.342)$ were identified between acute $(\mathrm{ES}=0.14$; $95 \% \mathrm{CI}=-0.07$ to 0.35 ; within-group $I^{2}=13.8 \%$, five study groups) and chronic effects ( $E S=0.01 ; 95 \% C I=-0.18$ to 0.19 ; within-group $I^{2}=0.0 \%$, one study group).

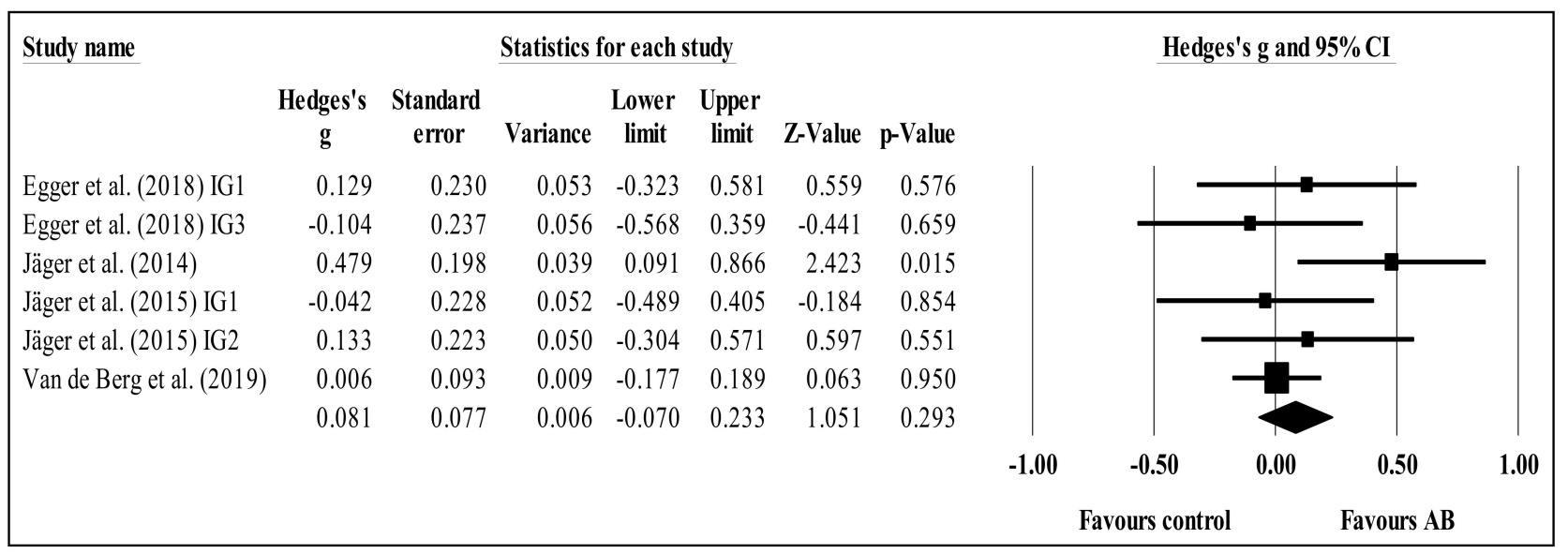

Figure 2. Forest plot of changes in inhibition in school-age students participating in active breaks (AB) compared to controls. Values shown are effect sizes (Hedges' g) with 95\% confidence intervals (CI). The size of the plotted squares reflects the statistical weight of each study. The black diamond reflects the overall result. This trend is not statistically significant. IG: intervention group.

\subsubsection{Effects on Concentration}

Eight studies reported an index of concentration performance, which has been mainly measured by the $\mathrm{d} 2$ and FACES tests. Regarding the acute effects, one of them found positive effects of 12-min VPA on 9 to 10-year-old students' concentration [44]. On the other hand, positive chronic effects after four and 10 weeks of intervention were found in two of four studies [46,48]; however, the four-week study of these was assessed as high risk at ROBINS-I [48]. No other significant effects were found.

Six studies provided data for concentration, involving nine experimental and six control groups (pooled $n=881$ ). There was a trivial effect of $\mathrm{AB}$ on concentration ( $\mathrm{ES}=0.19$; $95 \% \mathrm{CI}=-0.08$ to $0.46 ; p=0.161 ; I^{2}=63.5 \%$; Egger's test $p=0.581$; relative weight of each group: 8.2 to $17.9 \%$; Figure 3). A sensitivity analysis according to ROBINS-I was conducted, removing the study of Buchele et al. (2018) [48], with no significant changes in results. However, a study-by-study sensitivity analysis, removing the study of Schmidt et al., (2019) [24], revealed a small effect of $A B$ on concentration performance ( $E S=0.34 ; 95 \%$ CI $=0.20$ to $0.48 ; p<0.001)$. No significant sub-group differences $(p=0.627)$ were identified between acute ( $\mathrm{ES}=0.27 ; 95 \% \mathrm{CI}=-0.07$ to 0.60 ; within-group $I^{2}=0.0 \%$, four study groups) and chronic effects ( $E S=0.14 ; 95 \% C I=-0.29$ to 0.56 ; within-group $I^{2}=81.2 \%$, five study groups). 


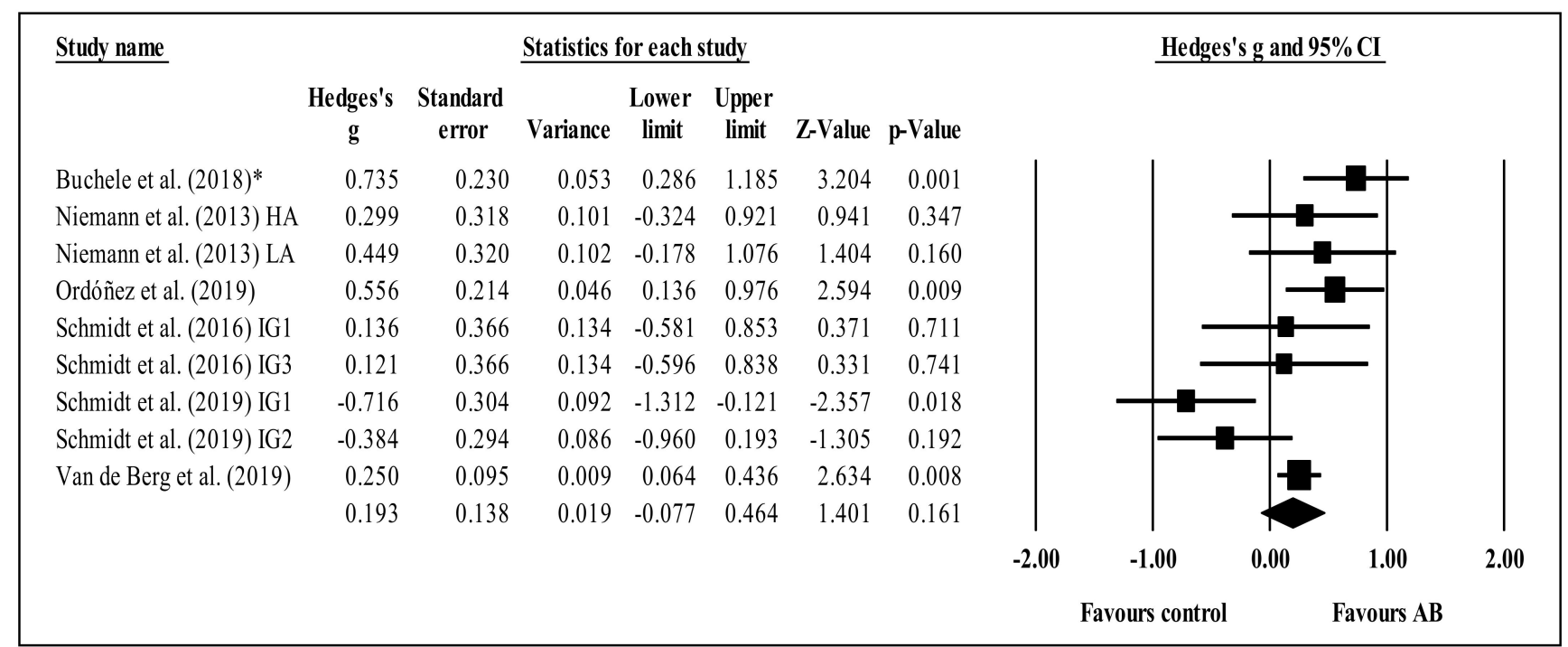

Figure 3. Forest plot of changes in concentration in school-age students participating in active breaks (AB) compared to controls. Values shown are effect sizes (Hedges' g) with 95\% confidence intervals (CI). The size of the plotted squares reflects the statistical weight of each study. The black diamond reflects the overall result. This trend is not statistically significant. IG: intervention group; HA: high-active subgroup; LA: low-active subgroup. * Critical risk in ROBINS-I.

\subsubsection{Effects on Selective Attention}

Six studies measured selective attention with the d2, FACES and the Sky-Search task in TEA-Ch tests. With the exception of one study [23], the other three studies that measured acute effects reported positive results, with ABs varying between 12 and 20 min of MPA or VPA. Of note, one of the interventions only reported favorable results on the group that participated in two ABs during the same morning and no differences between one $\mathrm{AB}$ and no $\mathrm{AB}$ [35], while other reported only benefits for the $\mathrm{AB}$ of $\mathrm{MPA}$ and not for the VPA AB [6]. In addition, greater benefits were reported among low-income students [45]. Regarding the chronic effects, results were similar to the concentration's results.

Four studies provided data for selective attention, involving five experimental and four control groups (pooled $n=395$ ). There was a moderate effect of AB on selective attention $\left(\mathrm{ES}=0.61 ; 95 \% \mathrm{CI}=0.41\right.$ to $0.82 ; p<0.001 ; I^{2}=0.0 \%$; Egger's test $p=0.036$ (corrected values: $\mathrm{ES}=0.67,95 \% \mathrm{CI} 0.45$ to 0.88 ); relative weight of each group: 7.2 to 41.1\%; Figure 4). A sensitivity analysis according to ROBINS-I was conducted, removing the study of Buchele et al. (2018) [48], with no significant changes in results (i.e., $p$-value remained at $<0.001$, mean ES $=0.59)$. Similarly, after study-by-study and group-by-group sensitivity analyses, no significant changes in results were noted (i.e., $p$-value remained at $<0.001$, mean ES $=0.48$ to 0.65$)$. No significant sub-group differences ( $p=0.963$ ) were identified between acute $\left(\mathrm{ES}=0.55 ; 95 \% \mathrm{CI}=0.10\right.$ to 1.00 ; within-group $I^{2}=43.5 \%$, three study groups) and chronic effects (ES $=0.54 ; 95 \% \mathrm{CI}=0.23$ to 0.84 ; within-group $I^{2}=0.0 \%$, two study groups). 


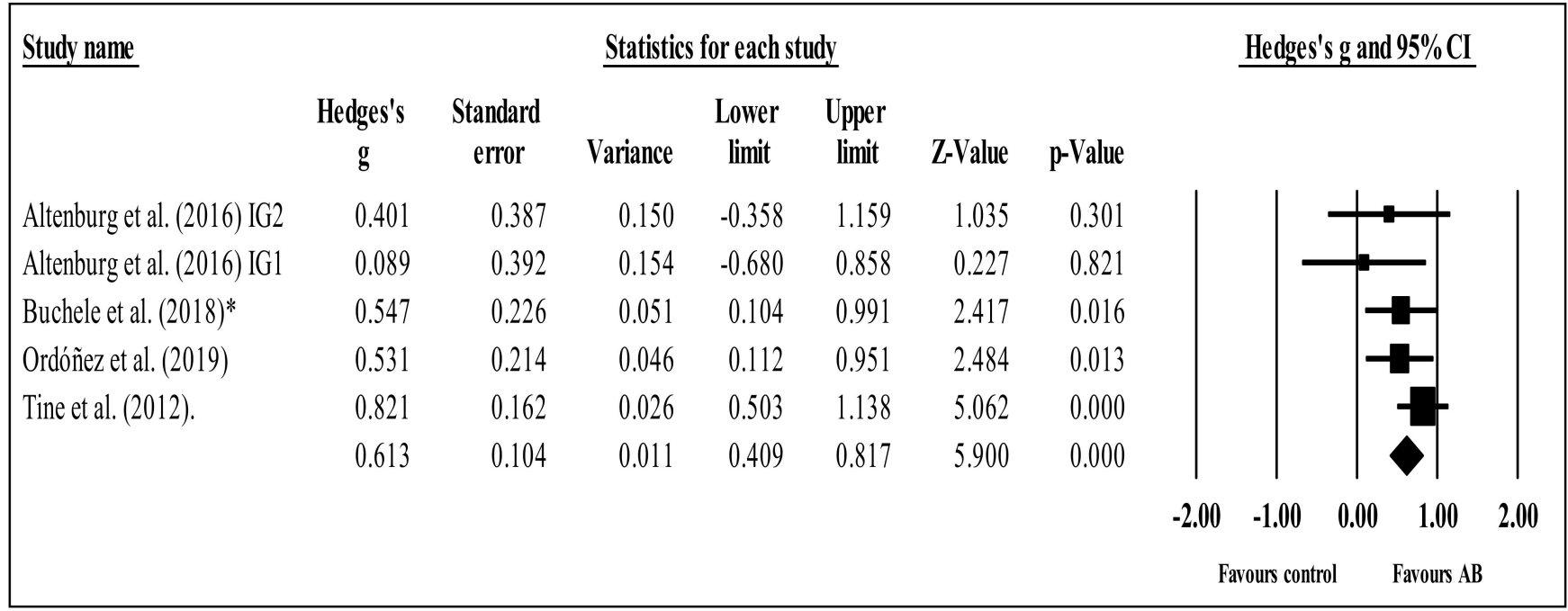

Figure 4. Forest plot of changes in selective attention in school-age students participating in active breaks (AB) compared to controls. Values shown are effect sizes (Hedges' g) with 95\% confidence intervals (CI). The size of the plotted squares reflects the statistical weight of each study. The black diamond reflects the overall result. This is a statistically significant result. IG: intervention group. * Critical risk in ROBINS-I.

\subsubsection{Effects on Shifting}

Shifting was only assessed in three studies [41-43] that used the flanker task to test acute effects. None of them found any acute effect after ABs. In the meta-analyses, the three studies provided data, involving five experimental and three control groups (pooled $n=441$ ). There was a trivial effect of $\mathrm{AB}$ on shifting ( $\mathrm{ES}=-0.18 ; 95 \% \mathrm{CI}=-0.52$ to 0.15 ; $p=0.286 ; I^{2}=65.7 \%$; Egger's test $p=0.229$; relative weight of each group: 19.1 to $21.8 \%$; Figure 5). After study-by-study and group-by-group sensitivity analyses, no significant changes in results were noted (i.e., $p$-value remained at $>0.05$, mean ES $=-0.29$ to 0.27 ).

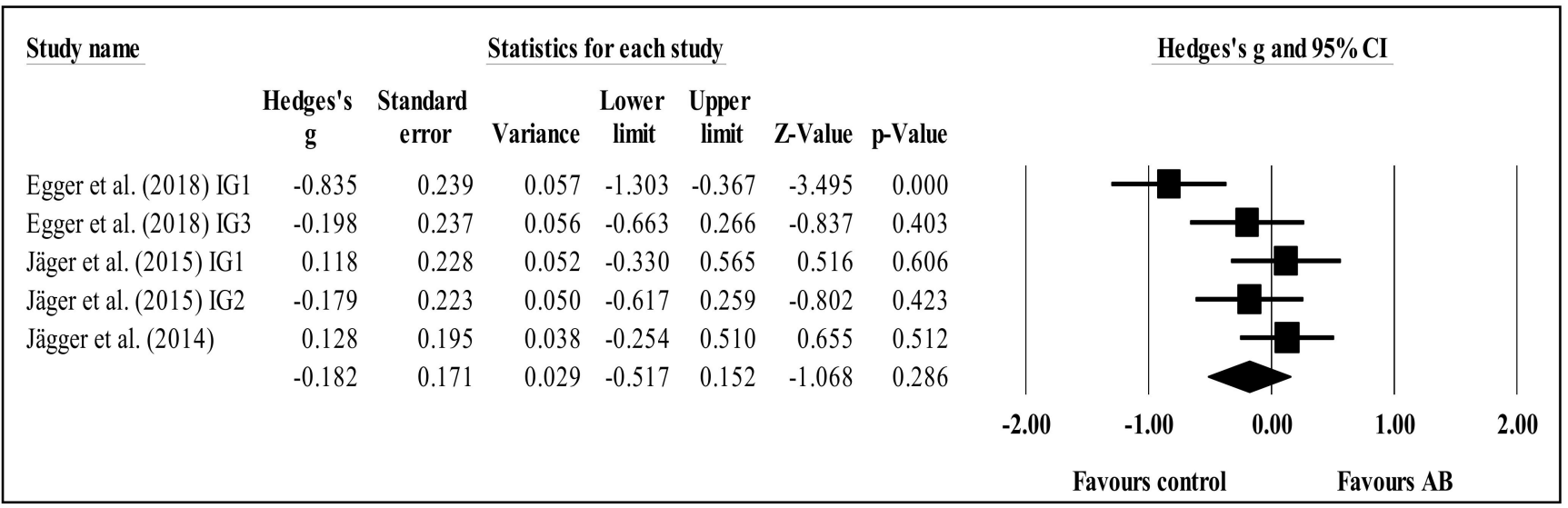

Figure 5. Forest plot of changes in shifting in school-age students participating in active breaks (AB) compared to controls. Values shown are effect sizes (Hedges's g) with 95\% confidence intervals (CI). The size of the plotted squares reflects the statistical weight of each study. The black diamond reflects the overall result. This trend is not statistically significant. IG: intervention group.

\subsubsection{Effects on Sustained Attention/Vigilance}

ANT, $\mathrm{d} 2$ and PVT tests were employed in each of the three studies that measured sustained attention/vigilance [47-49]. No acute effects were found [49]. On the other hand, positive chronic effects after a four-week intervention were found [48], but it presented 
a high risk at ROBINS-I. Meta-analyses could not be run since there were less than three studies per analysis.

\subsubsection{Effects on Other Outcomes}

Only one study [47] measured orienting, in 9 to 12-year-old students, before and after a 9-week intervention of daily MVPA cognitive ABs. No chronic effects were found. In addition, one study, of 8 to 11-year-old students, reported a global outcome of attention by a compendium of different executive function tasks [50]. This study found favorable acute effects after 10-15 min of AB involving aerobic MPA. Due to the reduced number of studies reporting data for these outcomes, a meta-analysis was precluded.

\section{Discussion}

\subsection{Discussion of Evidence}

The present systematic review with meta-analysis scrutinized and synthesized the literature related to the effects of ABs on students' (of any age) attention when compared to control conditions. The results do not point to any clear acute or chronic effects of $\mathrm{ABs}$ on students' overall attention, although some positive effects were found in terms of accuracy, concentration, inhibition, sustained attention, and (especially) selective attention. The meta-analysis revealed no statistical differences between $\mathrm{AB}$ and control groups regarding inhibition (Figure 2), concentration (Figure 3), or shifting (Figure 5). The trends for inhibition and concentration favored $\mathrm{AB}$ groups, and the trend for shifting favored the control groups. Nevertheless, all three meta-analyses included zero in their confidence intervals; therefore, no solid conclusions can be drawn. However, for selective attention, there was a significant difference between the $\mathrm{AB}$ and control groups (Figure 4) in favor of the former. These results will be discussed. In addition, overall, ABs did not compromise students' attention.

As a first approximation to the problem, we suggest that the small number of positive effects $[6,23,35,42,44-46,48,50]$ in the different included outcomes could be attributed to the fact that performing any type of exercise provokes neurophysiological changes in the brain [52]. Nevertheless, there is much heterogeneity and a wide variety of ABs protocols encountered (i.e., durations ranging from 4 to $20 \mathrm{~min}$; intensities of exercise ranging from moderate and vigorous; the inclusion of various types of PA such as aerobic, anaerobic, and muscular resistance; and the use of specific cognitive tasks to assess attention such as d2, ANT, and the flanker task). These differences do not provide clear evidence and have sparked controversy due to the non-existence of general guidelines for applying and implementing ABs.

Regarding the acute exercise paradigm, positive effects were observed only for accuracy [23], concentration [44], inhibition [42], and selective attention [6,35,45]. This suggests that cognitive activities performed after exercise lasting 4 to $20 \mathrm{~min}$ could produce overall benefits to students' attention. In addition, regarding the studies including chronic $A B$ interventions, positive effects were observed after 10 weeks in terms of concentration and selective attention [46]. Likewise, positive chronic effects on sustained attention were found after a four-week intervention [48].

Despite the lack of support from the meta-analyses, the positive findings found regarding selective attention are in line with the study of Donnelly et al. (2016) [53], who showed that routinely practicing PA in schools enhances cognitive performance. In fact, chronic exercise positively influences different attention processes in children [54]. In all cases, an argument could be made for the importance of studying additional moderators since these could influence the effects of exercise [20]. However, the small number of studies found per outcome did not allow us to make robust distinctions about the effects according to the moderators [20].

Despite the lack of clear moderators explaining the relationship between exercise and cognitive function, the results suggest that the intensity of the exercise used in ABs plays a fundamental role in the literature exploring the specific effects of PA on cognition. In fact, 
the current research suggests that some attentional outcomes improved after ABs at MVPA intensities (40 to 80 of $\mathrm{VO}_{2} \mathrm{max}$ ). However, many studies did not monitor the intensities of ABs, nor did they measure the magnitudes of the changes in some physiological mechanisms (e.g., brain-derived neurotrophic factor, catecholamines, increased cerebral blood flow) $[20,55]$ to predict their possible effects on behavior and cognitive performance.

Nevertheless, in most studies, ABs were carried out in the classroom and never under laboratory conditions. For this reason, measuring exercise intensities with a large sample is a truly complex matter. In fact, objective instruments (either a heart rate monitor $[19,35,41-43,45,51]$ or accelerometry $[6,24,47,49])$ were used to calculate the loads of ABs. However, researchers have also relied on subjective measures controlled by the teachers-in some cases, the measures were simply not registered $[23,44,46,48,50]$. In all cases, a potential and valid proposal might be the use of the subjective perception of effort (e.g., Egger et al. 2018 [41], Schmidt et al. 2016 [19]). This approach, which helps calculate metabolic changes during exercise, could be an effective option to use in school children [56,57].

In light of the above discussion, another key factor that might moderate the effects of ABs is the person who applies the AB. In this sense, it would be appropriate for physical education teachers to be responsible for applying ABs in all interventions [1,27]. On the one hand, they have the capacity to guide research proposals since they have a deeper knowledge of training principles involved in any kind of PA. On the other hand, they could provide students and other teachers with techniques for controlling the intensity of the $A B$ in each intervention, which is suggested as being a determinant of outcomes in the present work [26].

\subsection{Study Limitations}

The first limitation of this systematic review is the small number of studies found per outcome and associated effect of PA (i.e., acute or chronic) and the heterogeneity among these studies' designs. This leads to the second main limitation, which is that the ESs for each effect type cannot rely on a minimum of three studies in all cases. In addition, the heterogeneity was considerable for chronic effects in concentration and acute effects in shifting. Altogether, these limitations indicate that the results should be interpreted with caution.

\subsection{Practical Implications}

The outcomes of this study present implications for incorporating ABs into school lessons to improve students' attention. Through ABs students can reach higher levels of PA, which promotes a healthy lifestyle.

However, teachers are not usually adequately prepared to carry out ABs throughout the day during class. Thus, teachers should be trained on the correct implementation of ABs and the integration of movement on class days to ensure that ABs positively affect students' health and cognition. To achieve this, ABs should control physiological measures to objectively calculate exercise intensity. As a result, students might obtain more benefits from $\mathrm{ABs}$ if teachers are also trained in the use and interpretation of measures of PA intensity feasible for in-class use, such as the Borg scale [58-60].

Finally, as it relates to practical implications, the duration of ABs varied from 4 min to more than $20 \mathrm{~min}$. However, previous research has shown that only exercise of more than 20 min had positive results on cognitive performance [20]. From an educational point of view, it could be thought that adding a 20-min break into current school timetables may compromise the learning time. Therefore, ABs of such a length may not be practical. Additionally, evidence on this matter is not clear, as a recent review found that the duration of PA was inversely related to attentional performance [12].

Notwithstanding, from an academic performance perspective, increasing the amount of school-based PA does not compromise academic achievement and can improve classroom behavior and academic achievement [14]. In addition, as seen in some of the included 
studies, ABs can include academic content [24,49] and, therefore, could also be included in the learning time. Although more conclusive evidence is needed on this topic, ABs could be included and adapted to different educational contexts and would be effective for improving students' health and cognitive outcomes as long as the required intensity and time are met.

\section{Conclusions}

There are no clear positive effects of ABs on students' attention. The heterogeneity in the designs and measurements of the studies and the small number of studies carried out in school environments are the main reasons for the lack of conclusive results. Notwithstanding, it seems that including PA in school time through ABs does not compromise students' attention, and it could positively affect selective attention.

The intensity and duration of the PA seem to play a key role in cognitive effects. Therefore, efforts should be made to help teachers understand how to motivate their students to reach the correct intensity levels when carrying out an AB.

Even though research on ABs started around a decade ago, clear evidence is still lacking regarding their effects on attention. The results presented here highlight that this topic is still of significant relevance.

Author Contributions: Conceptualization, S.G.-V. and F.M.C.; methodology, Á.I.-P., F.T.G.-F., H.S. and F.M.C.; software and formal analysis, R.R.-C.; investigation, Á.I.-P. and F.T.G.-F.; resources, H.S., S.G.-V. and F.M.C.; data curation, Á.I.-P., F.T.G.-F. and H.S.; writing-original draft preparation, Á.I.-P., F.T.G.-F. and F.M.C.; writing-review and editing, Á.I.-P., A.F.S., F.T.G.-F., R.R.-C. and F.M.C.; supervision, A.F.S., S.G.-V. and F.M.C.; funding acquisition, Á.I.-P., H.S., F.T.G.-F., S.G.-V. and F.M.C. All authors have read and agreed to the published version of the manuscript.

Funding: This research is funded by the Spanish Ministry of Universities, grant number EST19/00498; and by Consejería de Educación, Cultura y Deportes de Castilla-La Mancha cofinanciados por el Fondo Europeo de Desarrollo Regional (FEDER), grant number: SBPLY/19/180501/000147. No other specific sources of funding were used to assist in the preparation of this article.

Acknowledgments: Álvaro Infantes-Paniagua gratefully acknowledges that his research is supported by the Spanish Ministry of Education, Culture and Sports (FPU16/00082). Francisco Tomás González Fernández gratefully acknowledges that he is supported by Beca de Movilidad Erasmus+ para recibir formación (PDI) Erasmus 2020-2021. Hugo Sarmento gratefully acknowledges the support of a Spanish government subproject 'Integration ways between qualitative and quantitative data, multiple case development, and synthesis review as main axis for an innovative future in physical activity and sports research (PGC2018-098742-B-C31) (Ministerio de Economía y Competitividad, Programa Estatal de Generación de Conocimiento y Fortalecimiento Científico y Tecnológico del Sistema I+D+i)', which is part of the coordinated project 'New approach of research in physical activity and sport from mixed methods perspective (NARPAS_MM) (SPGC201800 × 098742CV0)'. Filipe Manuel Clemente gratefully acknowledges that this work is also funded by Fundação para a Ciência e Tecnologia/Ministério da Ciência, Tecnologia e Ensino Superior through national funds and when applicable co-funded EU funds under the project UIDB/50008/2020.

Conflicts of Interest: The authors declare no conflict of interest. 


\section{Appendix A}

Table A1. Excluded full texts with reasons.

\begin{tabular}{|c|c|}
\hline Excluded References & Reason for Exclusion \\
\hline Adsiz et al. (2012) [61] & No $A B$ \\
\hline Amicone et al. (2018) [62] & No AB \\
\hline Bartholomew et al. (2018) [63] & No attention: Time on task \\
\hline Ben-Zeev et al. (2020) [64] & No AB: PE lesson \\
\hline Blasche et al. (2018) [65] & No attention \\
\hline Budde et al. (2008) [28] & No proper CG: PE lesson \\
\hline Chou et al. (2020) [66] & No AB: PE lesson \\
\hline Chrismas et al. (2019) [67] & No $A B$ \\
\hline Contreras et al. (2020) [68] & Not written in English. No control group \\
\hline Egger et al. (2019) [69] & $\begin{array}{c}\text { No proper CG: highly demanding cognitive } \\
\text { lesson }\end{array}$ \\
\hline Fenesi et al. (2018) [70] & $\begin{array}{c}\text { No attention: self-reported mind wandering } \\
\text { question }\end{array}$ \\
\hline Flippin et al. (2020) [71] & No AB \\
\hline Gonzalez et al. (2020) [72] & Not written in English \\
\hline Grieco et al. (2016) [73] & No attention: Time on task \\
\hline Howie et al. (2015) [74] & No attention \\
\hline Howie et al. (2014) [75] & No attention: Time on task \\
\hline Kubesch et al. (2009) [76] & No AB: PE lesson \\
\hline Mahar (2011) [77] & Review \\
\hline Mavilidi et al. (2020) [78] & No attention: Time on task \\
\hline Mazzoli et al. (2019) [79] & No AB \\
\hline McGowan et al. (2020) [80] & Laboratory \\
\hline Merriman et al. (2020) [81] & Report \\
\hline Miklós et al. (2020) [82] & Laboratory \\
\hline Napoli et al. (2005) [83] & No AB \\
\hline Niedermeier et al. (2020) [84] & $\begin{array}{l}\text { No pre-posttest on objective measure of } \\
\text { attention }\end{array}$ \\
\hline Ochoa et al. (2020) [85] & Not written in English \\
\hline Owen et al. (2018) [86] & No AB \\
\hline Pesce et al. (2013) [87] & No $A B$ \\
\hline Ruiz-Ariza et al. (2021) [88] & Not written in English \\
\hline Sánchez-López et al. (2015) [89] & Protocol \\
\hline Sugahara et al. (2018) [90] & No AB \\
\hline Tan et al. (2016) [4] & No attention: Time on task \\
\hline Vazou, et al. (2020) [91] & No attention: Observation \\
\hline Watson et al. (2017) [92] & Protocol \\
\hline Watson et al. (2019) [93] & No attention: Classroom behavior \\
\hline Webster et al. (2015) [94] & No attention: Time on task \\
\hline
\end{tabular}


Table A2. Qualitative assessment.

\begin{tabular}{|c|c|c|c|c|c|c|c|c|c|}
\hline Study & Outcome & D1a & D1b & D2 & D3 & D4 & D5 & DS & Overall \\
\hline Altenburg et al. (2016) [35] & All & $\mathrm{L}$ & - & $\mathrm{L}$ & $\mathrm{L}$ & SC & $\mathrm{SC}$ & - & SC \\
\hline Buchele et al. $(2018) *[48]$ & All & $\mathrm{C}$ & $\mathrm{L}$ & $S$ & $\mathrm{~L}$ & $\mathrm{NI}$ & $\mathrm{M}$ & $\mathrm{M}$ & $\mathrm{C}$ \\
\hline Egger et al. (2018) [41] & All & SC & - & $\mathrm{SC}$ & $\mathrm{L}$ & $\mathrm{L}$ & $\mathrm{SC}$ & - & SC \\
\hline Hill et al. (2010) [50] & All & $\mathrm{L}$ & $\mathrm{L}$ & $\mathrm{SC}$ & $\mathrm{L}$ & $\mathrm{L}$ & $\mathrm{SC}$ & SC & SC \\
\hline Jäger et al. (2014) [42] & All & $\mathrm{SC}$ & - & $\mathrm{L}$ & $\mathrm{L}$ & $\mathrm{L}$ & $\mathrm{SC}$ & - & SC \\
\hline Jäger et al. (2015) [43] & All & $\mathrm{SC}$ & - & $\mathrm{SC}$ & $\mathrm{L}$ & $\mathrm{L}$ & $\mathrm{H}$ & - & $\mathrm{H}$ \\
\hline Janssen et al. (2014) [6] & All & SC & - & $\mathrm{SC}$ & $\mathrm{H}$ & $\mathrm{L}$ & $\mathrm{H}$ & $\mathrm{H}$ & $\mathrm{H}$ \\
\hline Ma et al. (2015) [23] & All & $\mathrm{SC}$ & - & $\mathrm{SC}$ & $\mathrm{H}$ & $\mathrm{L}$ & $\mathrm{SC}$ & $\mathrm{SC}$ & $\mathrm{H}$ \\
\hline Niemann et al. (2013) [44] & All & $\mathrm{L}$ & - & $\mathrm{SC}$ & $\mathrm{H}$ & $\mathrm{L}$ & $\mathrm{H}$ & - & $\mathrm{H}$ \\
\hline Ordóñez et al. (2019) [46] & All & $\mathrm{L}$ & $\mathrm{L}$ & SC & $\mathrm{H}$ & $\mathrm{L}$ & $\mathrm{SC}$ & & $\mathrm{H}$ \\
\hline Schmidt et al. (2016) [19] & All & SC & - & $\mathrm{L}$ & $\mathrm{L}$ & $\mathrm{L}$ & $\mathrm{SC}$ & - & SC \\
\hline Schmidt et al. (2019) [24] & All & $\mathrm{SC}$ & $\mathrm{L}$ & $\mathrm{SC}$ & $\mathrm{L}$ & $\mathrm{L}$ & SC & - & SC \\
\hline \multirow{2}{*}{ Tine et al. (2012) [45] } & $\begin{array}{l}\text { Selective } \\
\text { attention }\end{array}$ & $\mathrm{SC}$ & - & SC & $\mathrm{L}$ & $\mathrm{L}$ & $\mathrm{H}$ & - & $\mathrm{H}$ \\
\hline & Accuracy & SC & - & $\mathrm{SC}$ & $\mathrm{H}$ & $\mathrm{L}$ & $\mathrm{H}$ & - & $\mathrm{H}$ \\
\hline van den Berg et al. (2016) [51] & All & $\mathrm{L}$ & $\mathrm{L}$ & $\mathrm{SC}$ & $\mathrm{L}$ & $\mathrm{L}$ & $\mathrm{SC}$ & $\mathrm{L}$ & SC \\
\hline \multirow[t]{2}{*}{ van den Berg et al. (2019) [47] } & $\begin{array}{l}\text { All but focused } \\
\text { attention }\end{array}$ & $\mathrm{L}$ & $\mathrm{L}$ & SC & $\mathrm{L}$ & $\mathrm{L}$ & $\mathrm{SC}$ & - & SC \\
\hline & $\begin{array}{l}\text { Focused attention } \\
\text { (concentration) }\end{array}$ & $\mathrm{L}$ & $\mathrm{L}$ & SC & $\mathrm{L}$ & $\mathrm{H}$ & SC & - & $\mathrm{H}$ \\
\hline Wilson et al. (2016) [49] & All & $\mathrm{L}$ & $\mathrm{L}$ & $\mathrm{L}$ & $\mathrm{L}$ & $\mathrm{L}$ & SC & SC & SC \\
\hline
\end{tabular}

D1a: Randomization process; D1b: The timing of identification or recruitment of participants in a cluster-randomized trial; D2: Deviations from the intended interventions; D3: Missing outcome data; D4: Measurement of the outcome; D5: Selection of the reported result; DS: Period and carryover effects. C: Critical; H: High risk; L: Low risk; M: Moderate; NI: No information; S: Serious; SC: Some concerns. * Assessment from ROBINS-I: D1a: Bias due to confounding; D1b: Bias in selection of participants into the study; D2: Bias in classification of interventions; D3: Bias due to deviations from intended interventions; D4: Bias due to missing data; D5: Bias in measurement of outcomes; $\mathrm{S}$ : Bias in selection of the reported result.

Table A3. Protocols of interventions.

\begin{tabular}{|c|c|c|}
\hline Attentional Outcome & Test/Task & Calculation \\
\hline Accuracy & $\mathrm{d} 2$ & Errors $(\%)$ \\
\hline \multirow{2}{*}{ Concentration } & FACES & Total correct responses-Errors (Commission) \\
\hline & $\mathrm{d} 2$ & Total correct responses-Commission errors \\
\hline Global attention & $\begin{array}{l}\text { Compendium of tasks (paced serial addition, } \\
\text { size ordering, listening span, digit-span } \\
\text { backwards, and digit-symbol encoding) }\end{array}$ & Overall score \\
\hline \multirow{3}{*}{ Inhibition } & ANT (flanker) & Incongruent-Congruent \\
\hline & Flanker & Incongruent-Congruent \\
\hline & Stroop & Incongruent-Congruent \\
\hline Orienting & ANT (flanker) & Center cue-Spatial cue \\
\hline \multirow{3}{*}{ Selective attention } & $\mathrm{d} 2$ & Total number of responses-Errors \\
\hline & FACES & Total of right responses \\
\hline & TEA-Ch & $\begin{array}{l}\text { Time of pair identification-Time of motor } \\
\text { performance }\end{array}$ \\
\hline Shifting & Flanker & SCORE (Mixed block)-SCORE (Standard block) \\
\hline \multirow{3}{*}{ Sustained attention/Vigilance } & ANT (flanker) & SCORE no cue - SCORE center cue \\
\hline & $\mathrm{d} 2$ & Fluctuation rate \\
\hline & PVT & $\begin{array}{c}\text { Mean response time to a repeating visual } \\
\text { stimulus }\end{array}$ \\
\hline
\end{tabular}




\section{References}

1. Daly-Smith, A.J.; Zwolinsky, S.; McKenna, J.; Tomporowski, P.D.; Defeyter, M.A.; Manley, A. Systematic review of acute physically active learning and classroom movement breaks on children's physical activity, cognition, academic performance and classroom behaviour: Understanding critical design features. BMJ Open Sport Exerc. Med. 2018, 4, e000341. [CrossRef] [PubMed]

2. Masini, A.; Marini, S.; Gori, D.; Leoni, E.; Rochira, A.; Dallolio, L. Evaluation of school-based interventions of active breaks in primary schools: A systematic review and meta-analysis. J. Sci. Med. Sport 2020, 23, 377-384. [CrossRef] [PubMed]

3. Watson, A.; Timperio, A.; Brown, H.; Best, K.; Hesketh, K.D. Effect of classroom-based physical activity interventions on academic and physical activity outcomes: A systematic review and meta-analysis. Int. J. Behav. Nutr. Phys. Act. 2017, 14, 114. [CrossRef] [PubMed]

4. Goh, T.L.; Hannon, J.; Webster, C.; Podlog, L.; Newton, M. Effects of a TAKE 10! Classroom-Based Physical Activity Intervention on Third- to Fifth-Grade Children's On-task Behavior. J. Phys. Act. Health 2016, 13, 712-718. [CrossRef] [PubMed]

5. van den Berg, V.; Salimi, R.; de Groot, R.; Jolles, J.; Chinapaw, M.; Singh, A. “It's a Battle ... You Want to Do It, but How Will You Get It Done?": Teachers' and Principals' Perceptions of Implementing Additional Physical activity in School for Academic Performance. Int. J. Environ. Res. Public Health 2017, 14, 1160. [CrossRef]

6. Janssen, M.; Chinapaw, M.J.M.; Rauh, S.P.; Toussaint, H.M.; van Mechelen, W.; Verhagen, E.A.L.M. A short physical activity break from cognitive tasks increases selective attention in primary school children aged 10-11. Ment. Health Phys. Act. 2014, 7, 129-134. [CrossRef]

7. Muñoz-Parreño, J.A.; Belando-Pedreño, N.; Torres-Luque, G.; Valero-Valenzuela, A. Improvements in Physical Activity Levels after the Implementation of an Active-Break-Model-Based Program in a Primary School. Sustainability 2020, 12, 3592. [CrossRef]

8. Vazou, S.; Saint-Maurice, P.; Skrade, M.; Welk, G. Effect of Integrated Physical Activities with Mathematics on Objectively Assessed Physical Activity. Children 2018, 5, 140. [CrossRef]

9. Heward, W.L. Ten Faulty Notions About Teaching and Learning That Hinder the Effectiveness of Special Education. J. Spec. Educ. 2003, 36, 186-205. [CrossRef]

10. Guthold, R.; Stevens, G.A.; Riley, L.M.; Bull, F.C. Global trends in insufficient physical activity among adolescents: A pooled analysis of 298 population-based surveys with 1.6 million participants. Lancet Child Adolesc. Health 2020, 4, 23-35. [CrossRef]

11. Álvarez-Bueno, C.; Pesce, C.; Cavero-Redondo, I.; Sánchez-López, M.; Martínez-Hortelano, J.A.; Martínez-Vizcaíno, V. The Effect of Physical Activity Interventions on Children's Cognition and Metacognition: A Systematic Review and Meta-Analysis. J. Am. Acad. Child Adolesc. Psychiatry 2017, 56, 729-738. [CrossRef]

12. Haverkamp, B.F.; Wiersma, R.; Vertessen, K.; van Ewijk, H.; Oosterlaan, J.; Hartman, E. Effects of physical activity interventions on cognitive outcomes and academic performance in adolescents and young adults: A meta-analysis. J. Sports Sci. 2020, 38, 2637-2660. [CrossRef] [PubMed]

13. de Greeff, J.W.; Bosker, R.J.; Oosterlaan, J.; Visscher, C.; Hartman, E. Effects of physical activity on executive functions, attention and academic performance in preadolescent children: A meta-analysis. J. Sci. Med. Sport 2018, 21, 501-507. [CrossRef]

14. Álvarez-Bueno, C.; Pesce, C.; Cavero-Redondo, I.; Sánchez-López, M.; Garrido-Miguel, M.; Martínez-Vizcaíno, V. Academic Achievement and Physical Activity: A Meta-analysis. Pediatrics 2017, 140, e20171498. [CrossRef] [PubMed]

15. Stadler, M.A. Role of attention in implicit learning. J. Exp. Psychol. Learn. Mem. Cognit. 1995, 21, 674-685. [CrossRef]

16. Steinmayr, R.; Ziegler, M.; Träuble, B. Do intelligence and sustained attention interact in predicting academic achievement? Learn. Individ. Differ. 2010, 20, 14-18. [CrossRef]

17. Janssen, M.; Toussaint, H.M.; van Mechelen, W.; Verhagen, E.A. Effects of acute bouts of physical activity on children's attention: A systematic review of the literature. SpringerPlus 2014, 3, 410. [CrossRef] [PubMed]

18. Kremer, J.M.; Moran, A.; Walker, G.; Craig, C. Key Concepts in Sport Psychology; Sage: Newcastle upon Tyne, UK, 2012. [CrossRef]

19. Schmidt, M.; Benzing, V.; Kamer, M. Classroom-Based Physical Activity Breaks and Children's Attention: Cognitive Engagement Works! Front. Psychol. 2016, 7. [CrossRef] [PubMed]

20. Chang, Y.K.; Labban, J.D.; Gapin, J.I.; Etnier, J.L. The effects of acute exercise on cognitive performance: A meta-analysis. Brain Res. 2012, 1453, 87-101. [CrossRef]

21. Etnier, J.L.; Nowell, P.M.; Landers, D.M.; Sibley, B.A. A meta-regression to examine the relationship between aerobic fitness and cognitive performance. Brain Res. Rev. 2006, 52, 119-130. [CrossRef] [PubMed]

22. Basso, J.C.; Suzuki, W.A. The Effects of Acute Exercise on Mood, Cognition, Neurophysiology, and Neurochemical Pathways: A Review. Brain Plast. 2017, 2, 127-152. [CrossRef] [PubMed]

23. Ma, J.K.; Le Mare, L.; Gurd, B.J. Four minutes of in-class high-intensity interval activity improves selective attention in 9-to 11-year olds. Appl. Physiol. Nutr. Metab. 2015, 40, 238-244. [CrossRef] [PubMed]

24. Schmidt, M.; Benzing, V.; Wallman-Jones, A.; Mavilidi, M.-F.; Lubans, D.R.; Paas, F. Embodied learning in the classroom: Effects on primary school children's attention and foreign language vocabulary learning. Psychol. Sport Exerc. 2019, 43, 45-54. [CrossRef]

25. van den Berg, V.; Saliasi, E.; Jolles, J.; de Groot, R.H.M.; Chinapaw, M.J.M.; Singh, A.S. Exercise of Varying Durations: No Acute Effects on Cognitive Performance in Adolescents. Front. Neurosci. 2018, 12. [CrossRef] [PubMed]

26. McMullen, J.; Kulinna, P.; Cothran, D. Chapter 5 Physical Activity Opportunities During the School Day: Classroom Teachers' Perceptions of Using Activity Breaks in the Classroom. J. Teach. Phys. Educ. 2014, 33, 511-527. [CrossRef] 
27. González, F.T.; González-Vúllora, S. Bases metodológicas desde la neuroeducación para el diseño-aplicación de los descansos activos (Methodological bases from neuroeducation for the design-application of active breaks). In Neuroeducación: Ayudando a Aprender Desde Las Evidencias Científicas; González-Víllora, S., Bodoque-Osma, A.R., Eds.; Morata: Madrid, Spain, 2021; pp. 105-128.

28. Budde, H.; Voelcker-Rehage, C.; Pietraßyk-Kendziorra, S.; Ribeiro, P.; Tidow, G. Acute coordinative exercise improves attentional performance in adolescents. Neurosci. Lett. 2008, 441, 219-223. [CrossRef]

29. Higgins, J.P.T.; Green, S. (Eds.) Cochrane Handbook for Systematic Reviews of Interventions 2.6 [Updated September 2006]; John Wiley \& Sons, Ltd: Chichester, UK, 2006.

30. Moher, D.; Liberati, A.; Tetzlaff, J.; Altman, D.G. Preferred Reporting Items for Systematic Reviews and Meta-Analyses: The PRISMA Statement. PLoS Med. 2009, 6, e1000097. [CrossRef]

31. Sterne, J.A.C.; Savović, J.; Page, M.J.; Elbers, R.G.; Blencowe, N.S.; Boutron, I.; Cates, C.J.; Cheng, H.-Y.; Corbett, M.S.; Eldridge, S.M.; et al. RoB 2: A revised tool for assessing risk of bias in randomised trials. BMJ 2019, 14898. [CrossRef]

32. Eldridge, S.; Campbell, M.K.; Campbell, M.J.; Drahota, A.K.; Giraudeau, B.; Reeves, B.C.; Siegfried, N.; Higgins, J.P. Revised Cochrane Risk of Bias Tool for Randomized Trials (RoB 2): Additional Considerations for Cluster-Randomized Trials (RoB 2 CRT); 2020. Available online: https://sites.google.com/site/riskofbiastool/welcome/rob-2-0-tool/rob-2-for-cluster-randomized-trials (accessed on 1 January 2021).

33. Higgins, J.P.T.; Savović, E.; Page, M.J.; Sterne, J.A.C. Revised Cochrane Risk of Bias Tool for Randomized Trials (RoB 2): Additional Considerations for Crossover Trials; 2020. Available online: https://sites.google.com/site/riskofbiastool/welcome/rob-2-0tool/rob-2-for-crossover-trials (accessed on 1 January 2021).

34. Sterne, J.A.; Hernán, M.A.; Reeves, B.C.; Savović, J.; Berkman, N.D.; Viswanathan, M.; Henry, D.; Altman, D.G.; Ansari, M.T.; Boutron, I.; et al. ROBINS-I: A tool for assessing risk of bias in non-randomised studies of interventions. BMJ 2016, i4919. [CrossRef]

35. Altenburg, T.M.; Chinapaw, M.J.M.; Singh, A.S. Effects of one versus two bouts of moderate intensity physical activity on selective attention during a school morning in Dutch primary schoolchildren: A randomized controlled trial. J. Sci. Med. Sport 2016, 19, 820-824. [CrossRef]

36. Hopkins, W.G.; Marshall, S.W.; Batterham, A.M.; Hanin, J. Progressive Statistics for Studies in Sports Medicine and Exercise Science. Med. Sci. Sport. Exerc. 2009, 41, 3-12. [CrossRef] [PubMed]

37. Higgins, J.P.T.; Thompson, S.G. Quantifying heterogeneity in a meta-analysis. Stat. Med. 2002, 21, 1539-1558. [CrossRef] [PubMed]

38. Egger, M.; Smith, G.D.; Schneider, M.; Minder, C. Bias in meta-analysis detected by a simple, graphical test. BMJ 1997, 315, 629-634. [CrossRef] [PubMed]

39. Duval, S.; Tweedie, R. Trim and Fill: A Simple Funnel-Plot-Based Method of Testing and Adjusting for Publication Bias in Meta-Analysis. Biometrics 2000, 56, 455-463. [CrossRef] [PubMed]

40. Shi, L.; Lin, L. The trim-and-fill method for publication bias: Practical guidelines and recommendations based on a large database of meta-analyses. Medicine (Baltimore) 2019, 98, e15987. [CrossRef]

41. Egger, F.; Conzelmann, A.; Schmidt, M. The effect of acute cognitively engaging physical activity breaks on children's executive functions: Too much of a good thing? Psychol. Sport Exerc. 2018, 36, 178-186. [CrossRef]

42. Jäger, K.; Schmidt, M.; Conzelmann, A.; Roebers, C.M. Cognitive and physiological effects of an acute physical activity intervention in elementary school children. Front. Psychol. 2014, 5, 1-11. [CrossRef]

43. Jäger, K.; Schmidt, M.; Conzelmann, A.; Roebers, C.M. The effects of qualitatively different acute physical activity interventions in real-world settings on executive functions in preadolescent children. Ment. Health Phys. Act. 2015, 9, 1-9. [CrossRef]

44. Niemann, C.; Wegner, M.; Voelcker-Rehage, C.; Holzweg, M.; Arafat, A.M.; Budde, H. Influence of acute and chronic physical activity on cognitive performance and saliva testosterone in preadolescent school children. Ment. Health Phys. Act. 2013, 6, 197-204. [CrossRef]

45. Tine, M.T.; Butler, A.G. Acute aerobic exercise impacts selective attention: An exceptional boost in lower-income children. Educ. Psychol. 2012, 32, 821-834. [CrossRef]

46. Ordóñez, A.F.; Polo, B.; Lorenzo, A.; Zhang, S. Effects of a School Physical Activity Intervention in Pre-adolescents. Apunt. Educ. Fís. Deport. 2019, 136, 49-61. [CrossRef]

47. van den Berg, V.; Saliasi, E.; de Groot, R.H.M.; Chinapaw, M.J.M.; Singh, A.S. Improving cognitive performance of 9-12 years old children: Just dance? A randomized controlled trial. Front. Psychol. 2019, 10, 1-14. [CrossRef]

48. Buchele Harris, H.; Cortina, K.S.; Templin, T.; Colabianchi, N.; Chen, W. Impact of Coordinated-Bilateral Physical Activities on Attention and Concentration in School-Aged Children. BioMed Res. Int. 2018, 2018. [CrossRef]

49. Wilson, A.N.; Olds, T.; Lushington, K.; Petkov, J.; Dollman, J. The impact of 10-minute activity breaks outside the classroom on male students' on-task behaviour and sustained attention: A randomised crossover design. Acta Paediatr. 2016, 105, e181-e188. [CrossRef]

50. Hill, L.; Williams, J.H.G.; Aucott, L.; Milne, J.; Thomson, J.; Greig, J.; Munro, V.; Mon-Williams, M. Exercising Attention within the Classroom. Dev. Med. Child Neurol. 2010, 52, 929-934. [CrossRef] [PubMed]

51. van den Berg, V.; Saliasi, E.; de Groot, R.H.M.; Jolles, J.; Chinapaw, M.J.M.; Singh, A.S. Physical activity in the school setting: Cognitive performance is not affected by three different types of acute exercise. Front. Psychol. 2016, 7, 1-9. [CrossRef] [PubMed] 
52. Park, S.; Etnier, J.L. Beneficial Effects of Acute Exercise on Executive Function in Adolescents. J. Phys. Act. Health 2019, 16, 423-429. [CrossRef] [PubMed]

53. Donnelly, J.E.; Hillman, C.H.; Castelli, D.; Etnier, J.L.; Lee, S.; Tomporowski, P.; Lambourne, K.; Szabo-Reed, A.N. Physical Activity, Fitness, Cognitive Function, and Academic Achievement in Children: A Systematic Review. Med. Sci. Sports Exerc. 2016, 48, 1197-1222. [CrossRef]

54. Xue, Y.; Yang, Y.; Huang, T. Effects of chronic exercise interventions on executive function among children and adolescents: A systematic review with meta-analysis. Br. J. Sports Med. 2019, 53, 1397-1404. [CrossRef]

55. Brisswalter, J.; Collardeau, M.; René, A. Effects of Acute Physical Exercise Characteristics on Cognitive Performance. Sport. Med. 2002, 32, 555-566. [CrossRef]

56. Groslambert, A. Validation of a Rating Scale of Perceived Exertion in Young Children. Int. J. Sports Med. 2001, 22, 116-119. [CrossRef]

57. Parfitt, G.; Shepherd, P.; Eston, R.G. Reliability of effort production using the children's CALER and BABE perceived exertion scales. J. Exerc. Sci. Fit. 2007, 4, 49-55.

58. Hortigüela Alcalá, D.; Hernando Garijo, A.; Pérez-Pueyo, Á.; Fernández-Río, J. Cooperative Learning and Students' Motivation, Social Interactions and Attitudes: Perspectives from Two Different Educational Stages. Sustainability 2019, 11, 7005. [CrossRef]

59. Yuretich, R.F.; Khan, S.A.; Leckie, R.M.; Clement, J.J. Active-Learning Methods to Improve Student Performance and Scientific Interest in a Large Introductory Oceanography Course. J. Geosci. Educ. 2001, 49, 111-119. [CrossRef]

60. Borg, G. Borg's Perceived Exertion and Pain Scales; Human Kinetics: Champaign, IL, USA, 1998.

61. Adsiz, E.; Dorak, F.; Ozsaker, M.; Vurgun, N. The influence of physical activity on attention in Turkish children. HealthMED 2012, 6, 1384-1389.

62. Amicone, G.; Petruccelli, I.; De Dominicis, S.; Gherardini, A.; Costantino, V.; Perucchini, P.; Bonaiuto, M. Green Breaks: The Restorative Effect of the School Environment's Green Areas on Children's Cognitive Performance. Front. Psychol. 2018, 9, 1579. [CrossRef] [PubMed]

63. Bartholomew, J.B.; Golaszewski, N.M.; Jowers, E.; Korinek, E.; Roberts, G.; Fall, A.; Vaughn, S. Active learning improves on-task behaviors in 4th grade children. Prev. Med. (Baltim) 2018, 111, 49-54. [CrossRef] [PubMed]

64. Ben-Zeev, T.; Hirsh, T.; Weiss, I.; Gornstein, M.; Okun, E. The Effects of High-intensity Functional Training (HIFT) on Spatial Learning, Visual Pattern Separation and Attention Span in Adolescents. Front. Behav. Neurosci. 2020, 14. [CrossRef]

65. Blasche, G.; Szabo, B.; Wagner-Menghin, M.; Ekmekcioglu, C.; Gollner, E. Comparison of rest-break interventions during a mentally demanding task. Stress Health 2018, 34, 629-638. [CrossRef] [PubMed]

66. Chou, C.-C.; Chen, K.-C.; Huang, M.-Y.; Tu, H.-Y.; Huang, C.-J. Can Movement Games Enhance Executive Function in Overweight Children? A Randomized Controlled Trial. J. Teach. Phys. Educ. 2020, 39, 527-535. [CrossRef]

67. Chrismas, B.C.R.; Taylor, L.; Cherif, A.; Sayegh, S.; Bailey, D.P. Breaking up prolonged sitting with moderate-intensity walking improves attention and executive function in Qatari females. PLoS ONE 2019, 14, e0219565. [CrossRef] [PubMed]

68. Contreras Jordan, O.R.; Leon, M.P.; Infantes-Paniagua, A.; Prieto-Ayuso, A. Effects of active breaks in the attention and concentration of Elementary School students. Rev. Interuniv. Form. Profr. 2020, 95, 145-160. [CrossRef]

69. Egger, F.; Benzing, V.; Conzelmann, A.; Schmidt, M. Boost your brain, while having a break! The effects of long-term cognitively engaging physical activity breaks on children's executive functions and academic achievement. PLoS ONE 2019, 14. [CrossRef] [PubMed]

70. Fenesi, B.; Lucibello, K.; Kim, J.A.; Heisz, J.J. Sweat So You Don't Forget: Exercise Breaks During a University Lecture Increase On-Task Attention and Learning. J. Appl. Res. Mem. Cognit. 2018, 7, 261-269. [CrossRef]

71. Flippin, M.; Clapham, E.D.; Tutwiler, M.S. Effects of using a variety of kinesthetic classroom equipment on elementary students on-task behaviour: A pilot study. Learn. Environ. Res. 2020. [CrossRef]

72. Gonzalez Fernandez, F.T.; Baena Morales, S.; Vila Blanch, M.; Garcia-Taibo, O. Chronic effects in cognition of actives-breaks. Sport. Tech. J. Sch. Sport Phys. Educ. Psychomot. 2020, 6, 488-502. [CrossRef]

73. Grieco, L.A.; Jowers, E.M.; Errisuriz, V.L.; Bartholomew, J.B. Physically active vs. sedentary academic lessons: A dose response study for elementary student time on task. Prev. Med. (Baltim) 2016, 89, 98-103. [CrossRef] [PubMed]

74. Howie, E.K.; Schatz, J.; Pate, R.R. Acute Effects of Classroom Exercise Breaks on Executive Function and Math Performance: A Dose-Response Study. Res. Q. Exerc. Sport 2015, 86, 217-224. [CrossRef]

75. Howie, E.K.; Beets, M.W.; Pate, R.R. Acute classroom exercise breaks improve on-task behavior in 4th and 5th grade students: A dose-response. Ment. Health Phys. Act. 2014, 7, 65-71. [CrossRef]

76. Kubesch, S.; Walk, L.; Spitzer, M.; Kammer, T.; Lainburg, A.; Heim, R.; Hille, K. A 30-Minute Physical Education Program Improves Students' Executive Attention. Mind Brain Educ. 2009, 3, 235-242. [CrossRef]

77. Mahar, M.T. Impact of short bouts of physical activity on attention-to-task in elementary school children. Prev. Med. (Baltim) 2011, 52, S60-S64. [CrossRef]

78. Mavilidi, M.F.; Drew, R.; Morgan, P.J.; Lubans, D.R.; Schmidt, M.; Riley, N. Effects of different types of classroom physical activity breaks on children's on-task behaviour, academic achievement and cognition. Acta Paediatr. 2020, 109, 158-165. [CrossRef]

79. Mazzoli, E.; Teo, W.-P.; Salmon, J.; Pesce, C.; He, J.; Ben-Soussan, T.D.; Barnett, L.M. Associations of Class-Time Sitting, Stepping and Sit-to-Stand Transitions with Cognitive Functions and Brain Activity in Children. Int. J. Environ. Res. Public Health 2019, 16, 1482. [CrossRef] 
80. McGowan, A.L.; Ferguson, D.P.; Gerde, H.K.; Pfeiffer, K.A.; Pontifex, M.B. Preschoolers exhibit greater on-task behavior following physically active lessons on the approximate number system. Scand. J. Med. Sci. Sports 2020, 30, 1777-1786. [CrossRef]

81. Merriman, W.; González-Toro, C.M.; Cherubini, J. Physical Activity in the Classroom. Kappa Delta Pi Rec. 2020, 56, 164-169. [CrossRef]

82. Miklós, M.; Komáromy, D.; Futó, J.; Balázs, J. Acute Physical Activity, Executive Function, and Attention Performance in Children with Attention-Deficit Hyperactivity Disorder and Typically Developing Children: An Experimental Study. Int. J. Environ. Res. Public Health 2020, 17, 4071. [CrossRef] [PubMed]

83. Napoli, M.; Krech, P.R.; Holley, L.C. Mindfulness Training for Elementary School Students. J. Appl. Sch. Psychol. 2005, 21, 99-125. [CrossRef]

84. Niedermeier, M.; Weiss, E.M.; Steidl-Müller, L.; Burtscher, M.; Kopp, M. Acute Effects of a Short Bout of Physical Activity on Cognitive Function in Sport Students. Int. J. Environ. Res. Public Health 2020, 17, 3678. [CrossRef] [PubMed]

85. Ochoa Diaz, C.E.; Machado Maliza, M.E.; Guarneri Chacha, K.A. Active break strategy to improve students' attention to teaching activities. Rev. Conrado 2020, 16, 285-290.

86. Owen, K.B.; Parker, P.D.; Astell-Burt, T.; Lonsdale, C. Effects of physical activity and breaks on mathematics engagement in adolescents. J. Sci. Med. Sport 2018, 21, 63-68. [CrossRef]

87. Pesce, C.; Crova, C.; Marchetti, R.; Struzzolino, I.; Masci, I.; Vannozzi, G.; Forte, R. Searching for cognitively optimal challenge point in physical activity for children with typical and atypical motor development. Ment. Health Phys. Act. 2013, 6, 172-180. [CrossRef]

88. Ruiz-Ariza, A.; Lopez-Serrano, S.; Mezcua-Hidalgo, A.; Martinez-Lopez, E.J.; Abu-Helaiel, K. Acute effect of physically active rests on cognitive variables and creativity in Secondary Education. Retos Nuevas Tend. Educ. Fís. Deport. Recreacion 2021, 635-642.

89. Sánchez-López, M.; Pardo-Guijarro, M.J.; del Campo, D.G.-D.; Silva, P.; Martínez-Andrés, M.; Gulías-González, R.; DíezFernández, A.; Franquelo-Morales, P.; Martínez-Vizcaíno, V. Physical activity intervention (Movi-Kids) on improving academic achievement and adiposity in preschoolers with or without attention deficit hyperactivity disorder: Study protocol for a randomized controlled trial. Trials 2015, 16, 456. [CrossRef] [PubMed]

90. Sugahara, S.; Dellaportas, S. Bringing active learning into the accounting classroom. Meditari Account. Res. 2018, 26, 576-597. [CrossRef]

91. Vazou, S.; Long, K.; Lakes, K.D.; Whalen, N.L. “Walkabouts” Integrated Physical Activities from Preschool to Second Grade: Feasibility and Effect on Classroom Engagement. Child Youth Care Forum 2020. [CrossRef]

92. Watson, A.; Timperio, A.; Brown, H.; Hesketh, K.D. A primary school active break programme (ACTI-BREAK): Study protocol for a pilot cluster randomised controlled trial. Trials 2017, 18, 433. [CrossRef]

93. Watson, A.J.L.; Timperio, A.; Brown, H.; Hesketh, K.D. A pilot primary school active break program (ACTI-BREAK): Effects on academic and physical activity outcomes for students in Years 3 and 4. J. Sci. Med. Sport 2019, 22, 438-443. [CrossRef]

94. Webster, E.K.; Wadsworth, D.D.; Robinson, L.E. Preschoolers' Time On-Task and Physical Activity During a Classroom Activity Break. Pediatr. Exerc. Sci. 2015, 27, 160-167. [CrossRef] 